\title{
Scale genesis by dark matter and its gravitational wave signal
}

\author{
Zhaofeng Kang ${ }^{*}$ and Jiang Zhu๑ ${ }^{\dagger}$ \\ School of physics, Huazhong University of Science and Technology, Wuhan 430074, China
}

(Received 4 May 2020; accepted 10 September 2020; published 30 September 2020)

\begin{abstract}
Classical scale invariance (CSI) may shed light on the weak scale origin, but the realistic CSI extension to the standard model requires a bosonic trigger. We propose a scalar dark matter (DM) field $X$ as the trigger, establishing a strong connection between the successful radiative breaking of CSI and DM phenomenologies. The latter forces the breaking scale to approximately $\mathcal{O}(\mathrm{TeV})$. It brightens the test prospect of this scenario via a gravitational wave, a sharp prediction of CSI phase transition (CSIPT), which is first order and has strong supercooling. Moreover, we carefully deal with some techniques which are commonly used to analyze CSIPT but may be missed. In particular, we clarify the imprecision of Witten's formula used in the single field case to calculate the bubble nucleation rate and stress that the essence of Witten's approximation is the validity of high-temperature expansion.
\end{abstract}

DOI: 10.1103/PhysRevD.102.053011

\section{INTRODUCTION}

The origin and stabilization of the weak scale are the fundamental questions of the particle standard model (SM), and they lead the progresses of modern particle physics. Classical scale invariance (CSI) may shed light on these questions. The SM just contains two scales: the explicit negative mass parameter $-\mu^{2}|H|^{2}$ in the Higgs potential, which accounts for the weak scale origin, and the dynamical scale $\Lambda_{\mathrm{QCD}}$, which determines the mass scale of the composite particles such as proton and neutron. Sending $\mu^{2} \rightarrow 0$, the SM becomes scale invariant at the classical level. However, CSI is violated at quantum level by anomaly, so hopefully a scale can be radiatively generated 1]]. Moreover, CSI may be the symmetry that protects the weak scale free of the notorious fine-tuning, as long as the SM Higgs field does not couple to a heavy field with a sizeable strength, which generates a physical large quadratic correction to the Higgs field [2].

Nevertheless, the SM itself is not consistent with this symmetry, owing to the heaviness of top quark and the lightness of Higgs boson. To get a viable SM extension consistent with CSI, new elements, probably bosons, should be introduced to trigger CSI spontaneously breaking (CSISB) at the quantum level. The trigger is characterized by a relatively strong interaction with the scalon field, whose

\footnotetext{
zhaofengkang@gmail.com

jackpotzhujiang@gmail.com
}

Published by the American Physical Society under the terms of the Creative Commons Attribution 4.0 International license. Further distribution of this work must maintain attribution to the author(s) and the published article's title, journal citation, and DOI. Funded by SCOAP. vacuum expected value (VEV) is the main order parameter of CSISB. It is tempting to conjecture that the new element is the missing piece of the SM, the dark matter (DM). Put it in another more inspiring way, DM spin is related to the weak scale origin. In such a framework, DM plays a vital role, and it might "explain" why DM should be there. In turn, due to the CSI, DM mass is no longer a dimensional parameter put in by hand; instead, its mass origin is tied with CSISB. Moreover, as the trigger, the main interaction of DM should be with the scalon feld. Hence, this framework has its theoretical merits, being a basis for DM model building. Actually, studying DM in the CSI framework is not rare, and an incomplete list of references is collected in Refs. [3-14]. We then consider a simple CSI extension to SM by two real singlet scalars, with one being the scalon and the other one being the trigger, which is the DM candidate at the same time. DM playing the role of the trigger is very expected from DM relic density because the interactions of DM must be dominated by DM-scalon portal coupling so as to avoid the strong exclusion to the usual DM-Higgs portal coupling.

In the presence of multiscalars, one should carefully study the mechanisms of CSISB and electroweak spontaneously breaking. Different mechanisms are suitable for different parameter space of the dimensionless couplings; we refer to a nice review in Ref. [15]. Confronting the correct DM relic density and suppressed DM-nucleon recoil rate, we focus on two frequently studied cases:

(i) In Gildener-Weinberg approach on the valley, the tree-level potential admits a valley at some scale $\mu_{\mathrm{GW}}$, and one can apply the Gildener-Weinberg approach [16] to study CSISB. This approach treats the CSISB and the electroweak symmetry breaking (EWSB) in a single step. 
(ii) In the Coleman-Weinberg approach in the decoupling limit, if the Higgs interactions with the scalon and trigger are irrelevantly small, CSISB by the scalon is reduced to the conventional single field case, and one can apply the Coleman-Weinberg (CW) approach [17], whereas the EWSB proceeds via the usual mechanism.

Both scenarios leave viable parameter spaces, with scalon VEV at the multi-TeV scale and a heavy DM at the $\mathrm{TeV}$ scale.

DM triggers CSISB at zero temperature, and back to the early Universe, it triggers the CSI phase transition (CSIPT). It is known to be first order and tends to have a large supercooling, so abundant gravitational waves (GWs) may be produced during CSIPT. This has been studied by many groups [18-26]. Specified to our model, due to the requirements from DM phenomenologies, the CSISB scale is at the multi-TeV scale, hence a CSIPT scale, by virtue of strong supercooling, from $\mathrm{GeV}$ to hundreds of $\mathrm{GeV}$, which falls in the sensitivity region of a space-based interferometer. Despite the clear physical picture, there are some unclear technique points in analyzing CSIPT. As one of the focuses, this article is devoted to clarifying these subtle aspects.

The paper is organized as follows. In Sec. II, we study CSISB by virtue of the DM field, and in the following section, DM phenomenology is analyzed. In Secs. IV and $\mathrm{V}$, we study in detail the CSI phase transition and the subsequent GW signals, respectively. Section VI contains the conclusions and discussions.

\section{SCALE GENESIS BY SCALAR DARK MATTER}

As stated in the Introduction, in the CSI extension to the $\mathrm{SM}$, a radiative CSISB trigger, a bosonic field providing the dominant quantum correction, is indispensable. One simple option is a spin-1 gauge boson from a gauge group [27], for instance, $U(1)_{B-L}$. It is tempting to consider that the missing part of the SM, the DM field $X$, does this job. We find that related scenarios are investigated before, in particular, a spin-1 DM [28-31], though the authors do not explicitly name the scenario. Reference [4] for the first time studied the possibility of a spin-0 DM being the trigger. Anyway, DM as a trigger establishes a close connection between DM and CSISB, and their interplay gives rise to interesting phenomenologies. In this section, we will first set up the model and then analyze in detail how the DM could trigger radiative CSISB.

\section{A. Simple (effective) model setup}

To build a realistic CSI model that could successfully accommodate the weak scale and also the Higgs boson, it is better to consider that the weak scale is not the main scale for CSISB. Otherwise, the model will hit the Landau pole at a very low scale without an elaborate arrangement of the model [32]. So, we will focus on the scenario that CSISB is dominated by a scalar field $S$. It is dubbed as the scalon field, and we will also term it "scalon" for short. As expected, the simplest extension with $S$ but without a trigger fails [33]. In this paper, we introduce a real singlet scalar DM as the trigger. The total Lagrangian of our model is

$\mathcal{L}_{\text {tot }}=\mathcal{L}_{\mathrm{SM}}($ no Higgs potential $)+K_{S, X}-V_{0}(H, S, X)$,

where $K_{S, X}$ collects the kinetic terms for $S$ and $X$ and $V_{0}$ is the tree-level potential,

$$
\begin{aligned}
V_{0}= & \lambda|H|^{4}+\frac{\lambda_{h s}}{2}|H|^{2} S^{2}+\frac{\lambda_{h x}}{2}|H|^{2} X^{2}+\frac{\lambda_{s x}}{4} S^{2} X^{2} \\
& +\frac{\lambda_{s}}{4} S^{4}+\frac{\lambda_{x}}{4} X^{4},
\end{aligned}
$$

where $H$ is the SM Higgs doublet and its neutral component $H_{0}$ is decomposed as $H_{0}=\frac{1}{\sqrt{2}}\left(\operatorname{Re} H_{0}+i \operatorname{Im} H_{0}\right)$. The successful radiative CSISB vitally relies on the scalontrigger/DM coupling with a sizable $\lambda_{s x}$. To stabilize $X$, we impose a $Z_{2}$ symmetry which only acts on the DM field: $X \rightarrow-X$. A similar model has been studied in Ref. [19,34], but we will widely extend their discussions. Actually, we prefer to explain the above model as an effective model for the CSI framework having a DM trigger, and one can complete it in many different ways, e.g., the model based on local $U(1)_{B-L}$ considered in Ref. [4].

Because of CSI, an accidental $Z_{2}^{\prime}$ acting on $S$ emerges, $S \rightarrow-S$. As a result, CSISB at the same time breaks $Z_{2}^{\prime}$. On the other hand, it is well known that the spontaneously breaking of a discrete symmetry would give rise to the domain wall problem. It is easily cured by the seesaw sector for neutrino masses,

$$
-\mathcal{L}_{N}=y_{N} \bar{\ell} H N+\frac{\lambda_{s n}}{2} S N^{2}+\text { H.c. }
$$

because it explicitly breaks $Z_{2}^{\prime}$. As a side comment, in order to generate neutrino masses in the framework of CSI, a specific singlet scalon field $S$ may be always required, no matter whether via the normal seesaw mechanism or via radiative mechanism $[13,14,35]$. To simplify the discussions, we assume that terms in the seesaw sector are irrelevant to CSISB, and it is true as long as $\lambda_{s n}$ is sufficiently small.

\section{B. CSI radiative symmetry breaking}

Now, we investigate CSISB in the model considered in the last subsection. The beautiful idea that generating a mass scale from a theory without an explicit mass scale is by Coleman and Weinberg in the celebrated paper from 
1973 [1]. Their basic observation is that CSI is anomalous and thus it is broken by quantum effects, hence generating a scale, equivalently driving the ground state of the system away from the origin. The first attempt is a scalar QED, a single scalar field (the scalon) charged under an Abelian gauge group with gauge coupling $g_{X}$. Then, given the hierarchy $1 \gg g_{X}^{4} \sim \lambda_{s}$ with $\lambda_{s}$ the self-coupling of the scalon, the model succeeds in radiative CSISB via tree-loop balance in the perturbative region. However, the situation becomes more complicated if the scalon is a combination of several fields. Later Gildener and Weinberg proposed a method $[16,36]$ to reduce this complicated problem to the single-field case, but it merely applies to the models whose couplings demonstrate a hierarchy such that the quantum effects only play a role near the flat direction of the potential. Since the two approaches work in different patterns of couplings, we will analyze radiative CSISB in the two scenarios separately. But before that, it is more illustrative to start from the general way to deal with radiative CSISB in the general situations, which applies to cases with more than two fields.

\section{General strategy for multifield potential}

To study the radiative symmetry breaking of the model, we should start from the effective potential for the three classical scalar fields $\vec{\phi}_{\mathrm{cl}}=\left(h_{\mathrm{cl}}, s_{\mathrm{cl}}, X_{\mathrm{cl}}\right)$ for $\operatorname{Re} H_{0}, S$, and $X$; for simplicity, the subscript will be dropped. However, to our purpose, the DM field is supposed to develop no VEV, which effectively reduces the three-dimension field space to two dimensions. ${ }^{1}$ Then, the tree-level effective potential is directly read from the tree-level potential Eq. (2.2),

$$
V^{(0)}(h, s)=\frac{1}{4} \lambda h^{4}+\frac{1}{4} \lambda_{s} s^{4}+\frac{1}{4} \lambda_{h s} h^{2} s^{2} .
$$

As the simplest two-scalar system, this potential is shared by a variety of models, despite of different triggers. The tree-level potential does not contain any explicit mass scale, so its minimum must be located at the origin.

As long as the quantum correction does not change the potential in the $X$ direction significantly, this assumption is correct. For example, we can let $\lambda_{h x}, \lambda_{s x}$ be a positive parameter or let $\lambda_{x} \gg \lambda_{s}$, which makes potential in the $X$ direction dominate by the tree-level CSI potential.

To study if the quantum effects could realize CSISB, we incorporate the one-loop corrections to $V^{(0)}$, which are encoded in the $\mathrm{CW}$ potential,

\footnotetext{
${ }^{1}$ One way to prevent $X$ from developing a VEV may be requiring $\lambda_{h x}>0$, since we will find that the viable parameter to make $S$ develop a VEV is $\lambda_{h s}<0$. But this is merely a sufficient condition, and we propose another way to guarantee the vanishing VEV for $X$. Anyway, in the scale invariance (SI) model, a field setting at the origin is generic while away from the origin needs more attention.
}

$V^{(1)}(h, s)=\frac{1}{64 \pi^{2}} \sum_{a} n_{a} m_{a}(h, s)^{4}\left[\log \frac{m_{a}(h, s)^{2}}{\mu^{2}}-C_{a}\right]$,

where $\mu$ is the renomalization scale. Index $a$ runs over all particles coupling to the classical backgrounds, and the species $a$ contains internal degrees of freedom $n_{a}$, concretely,

$$
\begin{aligned}
n_{W} & =6, \quad n_{Z}=3, \quad n_{h}=1, \quad n_{\mathrm{GSB}}=3, \\
n_{t} & =-12, \quad n_{s}=1, \quad n_{X}=1 .
\end{aligned}
$$

The subscripts successively denote the $W / Z$ boson, Higgs boson, Goldstone bosons (GSBs), top quark in the SM, and the scalon, trigger/DM beyond the SM. $C_{a}$ are constants, and in the $\overline{\mathrm{MS}}$ scheme, $C_{a}=\frac{5}{6}$ for a spin-1 field, while $C_{a}=\frac{3}{2}$ for a spin-0 or spin- $1 / 2$ field. Note that if the radiative symmetry breaking involves large couplings questionable in perturbativity one should apply the renormalization group (RG) approach to improve the one-loop CW potential.

Let us turn to the key ingredients in the CW potential, the background field-dependent masses $m_{a}$. Most of them have simple analytical expressions,

$$
\begin{aligned}
m_{\mathrm{GSB}}^{2} & =\lambda h^{2}+\frac{1}{2} \lambda_{h s} s^{2}, \quad m_{X}^{2}=\frac{\lambda_{h x}}{2} h^{2}+\frac{\lambda_{s x}}{2} s^{2} \\
m_{W}^{2} & =\frac{g^{2}}{4} h^{2}, \quad m_{Z}^{2}=\frac{g^{2}+g^{\prime 2}}{4} h^{2}, \quad m_{t}^{2}=\frac{y_{t}^{2}}{2} h^{2},
\end{aligned}
$$

where $g$ and $g^{\prime}$ are the gauge couplings for $S U(2)_{L} \times U(1)_{Y}$ and $y_{t}$ is the top quark Yukawa coupling. The three GSB modes in the SM will cause the gauge-dependent issue for the effective potential, but this issue is not of concern at least in the vacuum, because there $m_{G S B}=0$ and therefore they do not contribute to the effective potential. A consistent treatment may need a new gauge [37,38]; see a recent work employing this new gauge [39]. The SM physical Higgs boson mixes with the scalon, having mass squared matrix

$M_{h-s}^{2}=\left(\begin{array}{cc}m_{h h}^{2} & m_{h s}^{2} \\ m_{h s}^{2} & m_{s s}^{2}\end{array}\right)=\left(\begin{array}{cc}\frac{6 \lambda h^{2}+\lambda_{h s} s^{2}}{2} & \lambda_{h s} h s \\ \lambda_{h s} h s & \frac{\lambda_{h s} h^{2}+6 \lambda_{s} s^{2}}{2}\end{array}\right)$.

The eigenvalues of $M_{h-s}^{2}$ are given by

$m_{\phi_{ \pm}}^{2}=\frac{1}{2}\left[\operatorname{Tr} M_{h-s}^{2} \pm \sqrt{\left(\operatorname{Tr} M_{h-s}^{2}\right)^{2}-4 \operatorname{Det} M_{h-s}^{2}}\right]$,

and the lighter one $\phi_{-}$is massless in the Gilender-Weinberg scenario.

In principle, to determine if the model realizes radiative CSISB, one can use brute force to search the ground state of 
the general $V^{(0)}+V^{(1)}$ without special structure; the vacuum is labeled by the VEV of the classical backgrounds $\left(v_{h}, v_{s}\right)$. But in practice, this approach is useful only in the context of numerical studies [15,39]. In some scenarios, discussed subsequently, the general potential can be reduced to the one-dimensional case where one can develop analytical understanding in the radiative CSISB.

After finding the vacuum, the particle spectra of the Higgs-scalon system can be obtained from the mass squared matrix Eq. (2.8) with $(h, s)$ replaced by $\left(v_{h}, v_{s}\right)$. But it just gives the leading-order result. This matrix receives substantial quantum corrections. In our studies, due to the large separation between the CSISB scale and weak scale, the corrections mainly come from the triggerDM coupling, so it is sufficient to just incorporate the loop correction to the scalon mass element. Quantum corrections may obviously modify the tree-level masses and mixing. It has immediate implications to the physics that are sensitive to the mixing angle, for instance, DMnucleon recoil. The spectra are characterized by the presence of another light $C P$-even Higgs boson different than the SM-like Higgs boson $h_{\mathrm{SM}}$, corresponding to the pseudo-GSB (pGSB) of the spontaneously breaking of anomalous CSI. Nevertheless, it is not bound to be the lighter one, and in principle, $h_{\mathrm{SM}}$ can be identified either with the heavier one $\phi_{+}$or the lighter one $\phi_{-}$.

\section{Higgs portal scenario by the $C W$ approach}

Let us first consider the scenario characterized by $0<-\lambda_{h s} \ll 1$. Then, it is justified to switch the Higgs portal coupling $\lambda_{s h}$ and study radiative CSISB just in the dark sector, which just contains the scalon $S$ and the trigger $X$ described by the following potential,

$$
V_{D S}=\frac{\lambda_{s}}{4} S^{4}+\frac{\lambda_{s x}}{4} S^{2} X^{2}+\frac{\lambda_{x}}{4} X^{4}
$$

$X$ should have no VEV, servicing as a spin-0 similarity to the gauge trigger in the original $\mathrm{CW}$ mechanism. One may ask why $S$ instead of $X$, which actually has similar couplings to $S$, is selected out as the scalon field, and in Appendix A, we give a short comment. Anyway, to study CSISB, the model is reduced to one single field case, and the tree-level potential of the scalon field is $V_{s}^{(0)}(s)=\frac{1}{4} \lambda_{s} s^{4}$.

Although not very necessary, we adopt the RG improved potential to incorporate quantum effects. This approach helps us determine the vacuum reliable in the perturbative region radiatively and also to manifest the difference between a spin- 0 and a gauge trigger. In general, the renormalization group equation (RGE) improved effective potential takes the form of [40]

$$
V_{s}^{(1)}(s)=\frac{1}{4} \lambda_{s}\left(t_{s}\right) G\left(t_{s}\right) s^{4}
$$

The quantum effects encoded in the wave function factor $\left.G\left(t_{s}\right)=\exp \left[\int_{0}^{t_{s}} d t^{\prime} \gamma_{s}\left(t^{\prime}\right)\right)\right]$ merely give a subleading contribution: $t_{s}=\log \frac{s}{\mu_{0}}$ with $\mu_{0}$ the renormalization scale. As a matter of fact, here, this contribution vanishes because of the coincidentally vanishing one-loop anomalous dimension of the scalon field, $\gamma_{s}(t)=0 .{ }^{2}$ The major quantum effects are encoded in the running coupling $\lambda_{s}\left(t_{s}\right)$, which is the solution of a set of coupled RGEs, whose beta functions are (in the MS scheme) given by

$$
\begin{aligned}
& \beta_{\lambda_{s}}=\frac{9}{8 \pi^{2}} \lambda_{s}^{2}+\frac{1}{32 \pi^{2}} \lambda_{s x}^{2}, \\
& \beta_{\lambda_{s x}}=\frac{1}{4 \pi^{2}} \lambda_{s x}^{2}+\frac{3}{8 \pi^{2}} \lambda_{s} \lambda_{s x}+\frac{3}{8 \pi^{2}} \lambda_{x} \lambda_{s x}, \\
& \beta_{\lambda_{x}}=\frac{9}{8 \pi^{2}} \lambda_{x}^{2}+\frac{1}{32 \pi^{2}} \lambda_{s x}^{2} .
\end{aligned}
$$

Other contributions are suppressed by $\lambda_{h s}^{2} \ll 1$ in the Higgs portal limit.

The dark sector (2.10) is different than the scalar QED where the trigger-scalaon coupling, namely, the gauge coupling, is the only parameter in the trigger sector, and the corresponding RGEs admit an analytical solution. But Eq. (2.12) involves not only trigger-scalon coupling $\lambda_{s x}$ but also an additional coupling, the trigger/DM self-interaction $\lambda_{x}$. Moreover, the $\beta$ function of the trigger-scalon coupling receives a cross term $\lambda_{s} \lambda_{s x}$, which is absent in the scalar QED system. Consequently, Eq. (2.12) no longer has an analytic solution. In Appendix A, we approximate the above RGEs to the $\lambda_{s}-\lambda_{s x}$ system, thus admitting an analytical solution.

Fortunately, determination of the condition for radiative CSISB $d\left(V_{s}^{(1)}(s)\right) / d s=0$ does not need to solve RGEs. Taking $\mu_{0}$ at the vacuum $v_{s}$, the extremum condition is translated to an equation between $\beta_{\lambda_{s}}, \gamma_{s}$ if present and $\lambda_{s}$ at $t_{s}=0$ :

$$
\begin{aligned}
\left.\left(\beta_{\lambda_{s}}+4 \lambda_{s}\left(t_{s}\right)\right)\right|_{t_{s}=0}= & 0 \Rightarrow \lambda_{s}(0)+\frac{1}{128 \pi^{2}} \lambda_{s x}(0)^{2} \\
& +\frac{9}{32 \pi^{2}} \lambda_{s}(0)^{2}=0
\end{aligned}
$$

It is the phenomenon of dimensional transmutation; the scale $v_{s}$ is traded with a relation between dimensionless couplings. $\lambda_{s}(0)$ should be very small, thus the $\lambda_{s}(0)^{2}$ term negligible, leading to the relation between scalon selfcoupling and trigger-scalon coupling at $t_{s}=0$,

$$
\lambda_{s}(0) \approx-\frac{1}{128 \pi^{2}} \lambda_{s x}(0)^{2} .
$$

\footnotetext{
${ }^{2}$ It is understood by such a fact: The one-loop correction to the two-point function of $s$ merely receives contributions from the bubble diagrams, a result of the $Z_{2}^{\prime}$ symmetry.
} 
It reproduces the well-known tree-loop hierarchy, but it is $\lambda_{\text {tree }} \sim \lambda_{\text {loop }}^{2}$ rather than $\lambda_{\text {loop }}^{4}$ in a scalar QED theory. Turning on the Higgs portal coupling leads to EWSB, which does not proceed radiatively, but as in the usual SM via a negative mass parameter, $\mu_{h}^{2}=-\lambda_{h s} v_{s}^{2} / 2$. It is an accidental result of the negative Higgs portal coupling between the scalon and SM Higgs doublet. The resulting weak scale is expressed as

$$
v_{h}=\sqrt{\frac{\mu_{h}^{2}}{\lambda}}=\sqrt{\frac{-\lambda_{h s}}{2 \lambda}} v_{s}
$$

fixed to be $v_{h}=246 \mathrm{GeV}$.

After pinning down the vacuum, now we present the spectra. The heavy trigger or DM mass squared is $m_{X}^{2} \approx \frac{1}{2} \lambda_{s x} v_{s}^{2}$. In the strict Higgs portal limit, there is no mixing between the SM Higgs boson and scalon. Then, the former gets mass as usual in the SM, while the latter as a pGSB gains a mass purely from quantum effects,

$$
m_{s}^{2}=\frac{d^{2}}{d s^{2}}\left[V_{s}^{(1)}(s)\right]_{s=v_{s}}=b_{X} v_{s}^{2}
$$

with $b_{X} \approx \frac{1}{32 \pi^{2}} \lambda_{s x}(0)^{2}$, the main part of $\beta_{\lambda_{s}}$. But the Higgs portal coupling generates a small mixing between them, and we should calculate the spectra from the mass squared matrix (2.8), with $m_{s s}^{2} \rightarrow m_{s s}^{2}+b_{X} v_{s}^{2}$ capturing the dominant quantum effect. Usually, $m_{s}$ is a good approximation to the actual scalon mass even after taking into account the small mixing effect.

\section{Flat region by Gildener-Weinberg approach}

Let us move to the scenario characterized by $V^{(0)}\left(\phi_{i}\right) \gg$ $V^{(1)}\left(\phi_{i}\right)$, and then quantum corrections numerically do not matter except for the places in the field space where $V^{(0)} \approx 0$. In the CSI models, the valley or flat direction is such a kind of place. It is determined by the nontrivial solution to the extremum equation $d V^{(0)} /\left.d \phi_{i}\right|_{\mu_{\mathrm{GW}}}=0$, which, specific to our model, is given by

$$
\lambda h^{2}+\frac{1}{2} \lambda_{h s} s^{2}=0, \quad \lambda_{s} s^{2}+\frac{1}{2} \lambda_{h s} h^{2}=0 .
$$

Their solution is denoted as $\phi \vec{N}$, corresponding to lines of degenerate local minimum (thus flat), which points to a definite direction $\vec{N}$ in the filed space, but leaving the modulus $\phi$ free. Equation (2.17) admits nonzero solutions only for a special relation (flat direction relation) among the dimensionless couplings,

$$
\frac{h^{2}}{s^{2}}=-\frac{1}{2} \frac{\lambda_{h s}}{\lambda}=-2 \frac{\lambda_{s}}{\lambda_{h s}} \Rightarrow \lambda_{h s}=-2 \sqrt{\lambda \lambda_{s}}<0 .
$$

The flat direction relation can be regarded as a renormalization condition at the Gildener-Weinberg scale $\mu_{\mathrm{GW}}{ }^{3}$ Now, the flat direction is expressed as the following:

$$
\left(\begin{array}{c}
h \\
s
\end{array}\right)=\phi \vec{N}=\phi\left(\begin{array}{c}
\cos \alpha \\
\sin \alpha
\end{array}\right)=\frac{\phi}{\sqrt{\lambda^{\frac{1}{2}}+\lambda_{s}^{\frac{1}{2}}}}\left(\begin{array}{c}
\lambda_{s}^{\frac{1}{4}} \\
\lambda^{\frac{1}{4}}
\end{array}\right) .
$$

In particular, we are interested in small mixing $\alpha \rightarrow \pi / 2$, and therefore Eq. (2.18) implies the hierarchy

$$
0<\lambda_{s} \ll-\lambda_{h s} \ll \lambda
$$

Like in the Higgs portal scenario, again we need a very small and moreover negative $\lambda_{h s}$, but the underlying reasons are not the same.

The radiative correction will lift the flat direction and create a local minimum at some $\phi$. To see this, we rewrite the tree-level effective potential (2.4) plus the radiative correction along the flat direction in terms of $\phi$, i.e., reducing the potential to the one-dimensional case,

$$
V^{(0)}+V^{(1)}(\phi \vec{N})=A \phi^{4}+B \phi^{4} \log \frac{\phi^{2}}{\mu_{\mathrm{GW}}^{2}},
$$

where $A$ and $B$ are dimensionless loop functions defined as

$$
\begin{aligned}
& A=\frac{1}{64 \pi^{2}} \sum_{a} n_{a} m_{a}(\vec{N})^{4}\left[\log m_{a}(\vec{N})^{2}-C_{a}\right] \\
& B=\frac{1}{64 \pi^{2}} \sum_{a} n_{a} m_{a}(\vec{N})^{4}
\end{aligned}
$$

which only depend on the tree-level couplings. As expected, the tree-level potential vanishes along the flat direction. By finding the extremum of $V^{(0)}+V^{(1)}(\phi \vec{N})$, we know that the modulus is fixed to be at the position related to the Gildener-Weinberg scale as

$$
\log \frac{\langle\phi\rangle^{2}}{\mu_{\mathrm{GW}}^{2}}=-\frac{1}{2}-\frac{A}{B} .
$$

The weak scale and the scalon scale, respectively, are given by $v_{h}=\langle\phi\rangle \cos \alpha=246 \mathrm{GeV}$ and $v_{s}=\langle\phi\rangle \sin \alpha$.

Using the flat direction relation, we are ready to show that the Higgs-scalon mass squared matrix (2.8) presents the GSB (or the consequence of flat direction), $m_{h} \phi_{-}=0$.

\footnotetext{
${ }^{3}$ Gildener proved that flat direction relation is feasible via choosing $\mu_{\mathrm{GW}}$ in the RGE [36]: starting from a potential without a valley at a generic scale, it can flow to the one with a valley at $\mu_{\mathrm{GW}}$. Hence, the flat direction relation does not require finetuning of couplings. However, this procedure works only for the couplings not far off the flat direction relation; otherwise, RGEs fail in driving the couplings to satisfy the flat direction relation at some scale.
} 
And quantum corrections add a new piece $8 B\langle\phi\rangle^{2} \sin ^{2} \alpha$ to the $m_{s s}^{2}$ element. In the small mixing limit $\alpha \rightarrow \pi / 2$, it is just the mass squared of the pGSB (as a reminder, pGSB is not necessary $\left.\phi_{-}\right)$. Then, to guarantee that the extremum is a minimum, $B>0$ is required, and it means

$$
6 m_{W}^{4}+3 m_{Z}^{4}+m_{h}^{4}+m_{X}^{4}-12 m_{t}^{4}>0,
$$

which yields the lower bound on DM mass, $m_{X}>316.48 \mathrm{GeV}$. Since $\alpha \rightarrow \pi / 2$, the main component of pGSB comes from the singlet, while the SM Higgs boson takes up the dominant fraction of the SM-like Higgs boson $h_{\mathrm{SM}}$, whose mass squared neglecting a small shift from loop is well approximated by

$$
\begin{aligned}
m_{h_{\mathrm{SM}}}^{2} & \approx \operatorname{Tr}\left(M_{h-s}^{2}\right)=\left(3 \lambda+\frac{\lambda_{h s}}{2}\right) v_{h}^{2}+\left(3 \lambda_{s}+\frac{\lambda_{h s}}{2}\right) v_{s}^{2} \\
& =\left(2 \lambda-\lambda_{h s}\right) v_{h}^{2} .
\end{aligned}
$$

Since $\lambda_{h s} \ll \lambda$, the above expression basically is the same as the one predicted in the SM. The Higgs-scalon mixing angle $\theta$ coincides with $\alpha$ at tree level, but it may be subjected to strong radiative correction, in particular when $8 B\langle\phi\rangle^{2}$ is close to $m_{h_{\mathrm{SM}}}^{2}$.

\section{SCALAR DARK MATTER VIA THE SCALON PORTAL}

In this section, we focus on the other face of the trigger, the DM candidate. It mainly interacts with the scalon field (hence, scalon portal), which is supposed to determine the DM relic density. Its correct value $\Omega h^{2} \simeq 0.12$ limits the scale of CSISB. Moreover, the scalon-SM Higgs boson mixing is subjected to constraints from the null DM direct detention results. In a word, the trigger being a DM has strong impacts on radiative CSISB.

First of all, we collect the relevant terms for the DM dynamics. We introduce a more illustrative notation to label the eigenstates of $M_{h-s}^{2}$. Let $O(\theta)$ be the orthogonal matrix that diagonalizes the full $M_{h-s}^{2}$, and its mass eigenstates $\phi_{ \pm}$ are renamed as $H_{i}=\left(h_{\mathrm{SM}}, \mathcal{S}\right)$, related to the flavor states via

$h_{\mathrm{SM}}=\cos \theta h+\sin \theta s, \quad \mathcal{S}=\cos \theta s-\sin \theta h$.

Note that $h_{\mathrm{SM}}$ can be identified with $\phi_{+}$or $\phi_{-}$, depending on the relative size of diagonal elements of $M_{h-s}^{2}$ at loop level. Then, the relevant interactions in the mass basis are collected in the Lagrangian

$-\mathcal{L}_{X}=\frac{1}{4} X^{2} \mathcal{S}^{2}+\frac{A_{i}}{2} X^{2} H_{i}+\frac{\lambda_{i j}}{4} X^{2} H_{i} H_{j}+\frac{y_{i q}}{\sqrt{2}} H_{i} \bar{q} q$, where $A_{i}=\lambda_{h x} v_{h} O_{1 i}+\lambda_{s x} v_{s} O_{2 i}$ and $y_{i q}=y_{q} O_{1 i}$, with $O$ specified in Eq. (3.1).

\section{A. DM relic density via freeze-out: CSIPT scale not far above $\mathrm{TeV}$}

If $X$ is the unique DM component, we have to guarantee that its relic density is correctly produced. Assuming an ordinary thermal history for $X$, its relic density is determined by the usual freeze-out dynamics, which requires that $X$ should have an annihilation cross section times the relative velocity at the freeze-out epoch $\langle\sigma v\rangle \simeq 1 \mathrm{pb}$.

The complete list of DM annihilation channels is long, for instance, into the various SM species via the Higgs portal and also into a pair of scalons. But it is well known that DM direct detection, discussed in the following subsection, compels us to consider a very small coupling between $X$ and the SM Higgs doublet. Being subdominant of the SM Higgs portal greatly simplifies the dynamics of $\mathrm{DM}$ at the early Universe, and the dominant annihilation channel of DM is $X X \rightarrow \mathcal{S S}$. It has a cross section times velocity $^{4}$

$\left\langle\sigma_{X X} v\right\rangle \simeq \frac{\lambda_{s x}^{2}}{64 \pi} \frac{1}{m_{X}^{2}}=0.89 \mathrm{pb} \times\left(\frac{\lambda_{s x}}{2.0}\right)\left(\frac{3 \mathrm{TeV}}{v_{s}}\right)^{2}$,

where we have taken the limit $m_{X} \gg m_{\mathcal{S}}$. It is seen that the scale of $v_{s}$ cannot be very high confronting the perturbative bound on $\lambda_{s x}<\pi$, which in turn means that if the DM is able to gain the correct relic density, its mass cannot exceed $4.4 \mathrm{TeV}$.

A long comment deserves attention. The ordinary freezeout dynamics of DM may be violated by supercooling CSIPT (discussed in a later section). Before the CSIPT, all particles including DM are massless, so DM is tightly coupled to the plasma. DM just gains mass after CSIPT. But if it is strongly supercooled, it is possible that the phase transition (PT) completion temperature $T_{*}<T_{f} \sim m_{X} / 20$ with $T_{f}$ the estimation on the decoupling temperature of normal DM. This means that DM number density is not frozen at $T_{f}$ but at $T_{*}$, when DM gains a heavy mass much above the plasma temperature of approximately $T_{*}$, thus failing to enter the new plasma inside the bubble, which expands to occupy the space dwelling in the metalstable vacuum. However, if the freeze-out dynamics indeed fails is a complicated question. One reason is that the reheating after CSIPT probably will heat the Universe to a very high temperature and therefore DM may be thermalized again. A solid discussion is beyond the scope of this work, and we leave this very interesting topic to a specific publication. Here, we just assume that the ordinary freeze-out still works.

\footnotetext{
${ }^{4}$ As noticed in Refs. [4,6], for a fermionic DM, the common mass and annihilation dynamics from a single interaction can make the above estimation independent on $\lambda_{s x}$.
} 


\section{B. DM direct detection bounds}

The DM-nucleon elastic scattering is mediated by the Higgs bosons $H_{i}$, and they can be integrated out, generating the effective operators between DM and quarks, $a_{q} X^{2} \bar{q} q$ with

$a_{q}=\frac{y_{q}}{\sqrt{2}} \frac{A_{i}}{2} O_{1 i} \frac{1}{m_{H_{i}}^{2}}=m_{q} \sum_{i}\left(\frac{\lambda_{h x} O_{1 i}^{2}}{2 m_{H_{i}}^{2}}+\frac{v_{s}}{v_{h}} \frac{\lambda_{s x} O_{1 i} O_{2 i}}{2 m_{H_{i}}^{2}}\right)$,

where the first and second are the contributions from the Higgs portal and scalon portal, respectively. Then, the DMnucleon elastic scattering cross section is $\sigma_{\mathrm{SI}}=\frac{4}{\pi} \mu_{p}^{2} f_{p}^{2}$ [41], with

$$
f_{p}=\frac{m_{p}}{2 m_{X}} \sum_{q} \frac{a_{q}}{m_{q}} f_{T_{q}}^{(p)}
$$

where $\mu_{p} \approx m_{p}$ is the reduced mass and $f_{T_{q}}^{(p)}$ encode the nuclear factors. The direct detection upper bounds strongly limit the size of $A_{i} O_{1 i} / m_{H_{i}}^{2}$. In particular, the usual SM Higgs portal coupling $\lambda_{h x}$ must be highly suppressed. So, we can just retain the scalon portal contribution in $f_{p}$, to derive

$$
\begin{aligned}
f_{p} & \approx \lambda_{s x} \frac{m_{p}}{8 m_{X}} \frac{v_{s}}{v_{h}} \sin 2 \theta\left(\frac{1}{m_{h_{\mathrm{SM}}}^{2}}-\frac{1}{m_{\mathcal{S}}^{2}}\right) \Delta^{p} \\
& \approx \frac{\sqrt{\lambda_{s x}}}{2 \sqrt{2}} \frac{m_{p}}{v_{s}} \sin 2 \theta\left(\frac{1}{m_{h_{\mathrm{SM}}}^{2}}-\frac{1}{m_{\mathcal{S}}^{2}}\right) \Delta^{p}
\end{aligned}
$$

where, to get the final expression for $f_{p}$, we have used $\Delta^{p}=\sum_{q=u, d, s} f_{T_{q}}^{(p)}+\sum_{q=t, b, d} f_{T_{q}}^{(p)} \approx 0.35 \quad$ and $\quad m_{X}^{2} \approx$ $\lambda_{s x} v_{s}^{2} / 2$ as a result of the DM mass genesis from CSISB.

There are several limits of special attention in studying the DM direct detection bound. First is the degenerate limit $m_{h_{\mathrm{SM}}} \approx m_{\mathcal{S}}$, which leads to a cancellation in the scalon portal contribution to $a_{q}$, manifest in Eq. $(3.6)^{5}$; hence, a relatively larger mixing angle is tolerated, given a substantial degeneracy. But note that the Higgs portal contribution does not show cancellation. Second is the heavy scalon limit $m_{\mathcal{S}}^{2} \gg m_{h_{\mathrm{SM}}}^{2}$, and then its contribution is suppressed compared to the SM Higgs boson. In other words, the scalon portal effectively becomes the SM Higgs portal, and one cannot rely on a heavy scalon to suppress $\sigma_{\mathrm{SI}}$. It holds, of course, assuming that $m_{\mathcal{S}}^{2}$ and $\theta$ are totally

\footnotetext{
${ }^{5}$ This cancellation is not accident and not novel [4]. However, in the usual cases where all Higgs bosons gain masses from a tree-level potential, being more degenerate means the system is more mixed. In the SI setup, the scalon gets mass just at quantum level, allowing degeneracy without a large mixing angle.
}

independent. The third is the opposite limit with $m_{\mathcal{S}}^{2} \ll m_{h_{\mathrm{SM}}}^{2}$, which requires a small mixing angle to avoid the direct detection bound. To be more specific, considering a $\mathrm{TeV}$ scale DM, then we have the upper bound on the mixing angle,

$$
\begin{aligned}
\sin \theta \lesssim & 0.07 \times\left(\frac{m_{\mathcal{S}}}{100 \mathrm{GeV}}\right)\left(\frac{m_{X}}{1 \mathrm{TeV}}\right)\left(\frac{\sigma_{\mathrm{SI}}^{\text {upper }}}{10^{-9} \mathrm{pb}}\right)^{1 / 2} \\
& \times\left(\frac{0.35}{\Delta^{p}}\right) \mathrm{GeV}^{-1}
\end{aligned}
$$

where we have used the estimation $m_{\mathcal{S}}^{2} \sim 10^{-2} \lambda_{s x}^{2} v_{s}^{2}$, but it may show a sizable deviation by virtue of the mixing effect. In any case, heaviness helps to alleviate the stringent direct detection bound on the scalar DM [42].

\section{Interplay between DM and radiative CSISB: Numerical results}

Now, we numerically demonstrate the implications of the DM trigger to radiative CSISB, to figure out the viable parameter space giving both successful CSISB and DM. As argued, the Higgs portal coupling $\lambda_{h x}$ plays no dynamics roles, and moreover should be irrelevantly small, thus simply set to $10^{-3}$. Then, there are four parameters $\lambda_{h}$, $\lambda_{s}, \lambda_{h s}$, and $\lambda_{s x}$ relevant to DM or/and CSISB. However, only one is free because of the three additional requirements for the correct weak scale, Higgs boson mass, and DM relic density. The single free parameter makes the parameter exploration become easy. It is further restricted by two constraints: one is from the current LHC Higgs data, which sets the upper bound on the SM Higgs and scalon mixing angle $|\sin \theta|<0.44$ [39], and the other one is from DM direct detection experiments such as XENON1T and PandaXII [43], which sets an upper bound on $\sigma_{\mathrm{SI}}$ for a given DM mass.

\section{Higgs portal scenario}

We first investigate the allowed parameter space in the Higgs portal scenario, where $\lambda_{s x}$ is chosen as the free parameter. We find that, even taking into account the large quantum effect, the heavier eigenstate $\phi_{+}$in Eq. (2.9) cannot be identified with the SM-like Higgs boson. Let us briefly explain the reasons. To satisfy the conditions $m_{\phi_{+}}=125 \mathrm{GeV}$ and the correct DM relic density via Eq. (3.3), the viable region is $0<\lambda_{s x}<1.04$. However, one meets $m_{\phi_{-}}^{2}<0$ for $\lambda_{s x}<0.33$ and $|\sin \theta|>0.44$ for $0.33<\lambda_{s x}<1.04$. So, we have to identify the SM-like Higgs boson with the lighter one $\phi_{-}$. Then, for a given $\lambda_{s x}$, the solution of the equation $m_{\phi_{-}}=125 \mathrm{GeV}$ as a function of $\lambda_{h s}$ has two branches. One branch requires a large Higgsscalon coupling $\lambda_{h s}>\lambda_{s x}$. But recall that the Higgs portal limit requires a small $\lambda_{h s}$, so this branch is unacceptable, whereas the other branch $\lambda_{h s}<\lambda_{s x}$ gives successful 

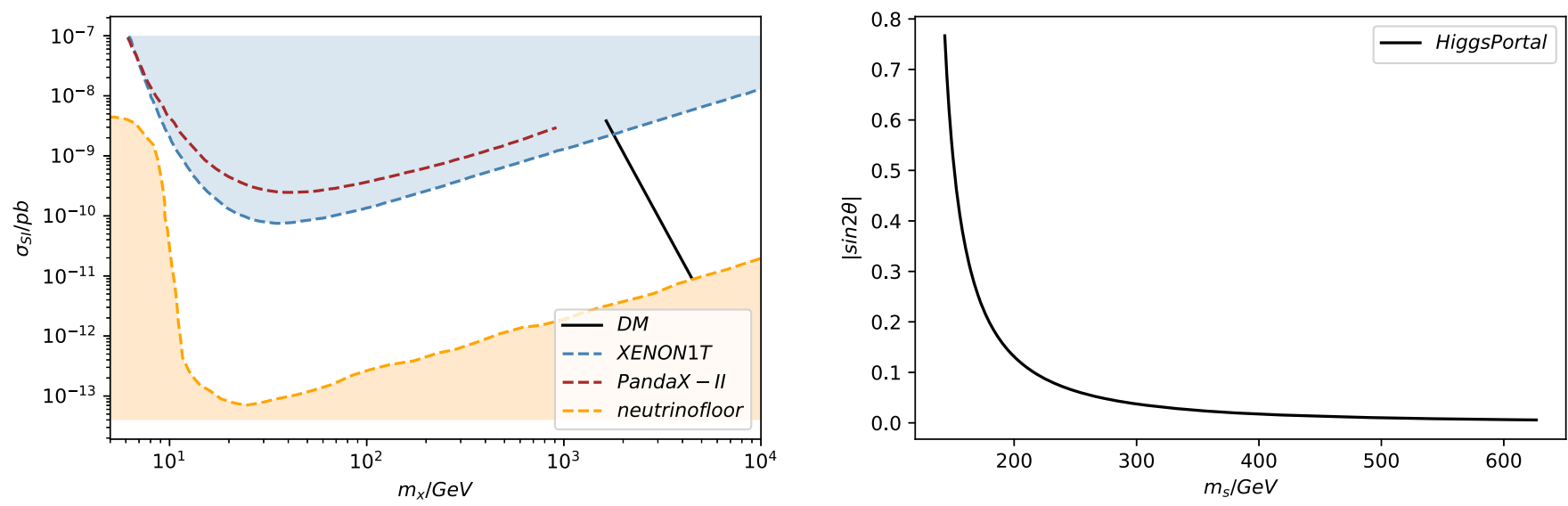

FIG. 1. Left: the surviving parameter space under the DM direct detection bound (black line) in the Higgs portal limit. Right: profile of scalon in the $m_{\mathcal{S}}-|\sin 2 \theta|$ plane for $0.46<\lambda_{s x}<\pi$.

phenomenology if $\lambda_{s x}$ is in the region $1.09<\lambda_{s x}<\pi$, with the upper bound for the sake of perturbativity. The condition $|\sin \theta|<0.44$ further shrinks the feasible parameter region to $1.16<\lambda_{s x}<\pi$.

Now, we add the stringent bound from DM direct detection. This scenario, by definition, should give a fairly small mixing angle. In the right panel of Fig. 1, we plot the profile of the scalon, on the $m_{\mathcal{S}}-\sin 2 \theta$ plane. One can see that $|\sin \theta| \lesssim \mathcal{O}\left(10^{-1}\right)$ as long as the scalon mass is not very close to $m_{h_{\mathrm{SM}}}$, which is good for evading the DM direct detection bound for a relatively heavy scalon, hence DM. This is just what the left panel of Fig. 1 shows; DM with mass $m_{X} \gtrsim 1.78 \mathrm{TeV}$ is still allowed, corresponding to $\lambda_{s x} \gtrsim 1.26$ and a scalon with mass $m_{\mathcal{S}} \gtrsim 160 \mathrm{GeV}$. In summary, in the Higgs portal scenario, the allowed parameter space lies in the interval

$$
1.26<\lambda_{s x}<\pi
$$

Moreover, the resulting scalon, the clear prediction of the CSI models, tends to be heavy and slightly mixed with the SM Higgs boson and thus is difficult to probe at the LHC.

\section{Gildener-Weinberg scenario}

Next, we study the Gildener-Weinberg scenario, where again $\lambda_{s x}$ is chosen as the free parameter. Then, we add the constraints step by step. First of all, the condition for a stable vacuum, namely, $B>0$ sets the lower bound $\lambda_{s x}>0.23$. Next, to identify the $125 \mathrm{GeV}$ SM-like Higgs boson with $\phi_{-}$or $\phi_{+}, \lambda_{s x}>1.08$ or $\lambda_{s x}<1.08$ is found. Finally, the upper bound on the mixing angle $|\sin 2 \theta|<0.79$ selects two regions $0.07<\lambda_{s x}<0.99$ or $1.13<\lambda_{s x}<\pi$. These constraints still allow a wide parameter space:

(i) $0.23<\lambda_{s x}<0.99$, where $h_{\mathrm{SM}}$ is identified with the heavier one $\phi_{+}$and DM mass is in the region $325 \mathrm{GeV}<m_{X}<1400 \mathrm{GeV}$.

(ii) $1.13<\lambda_{s x}<\pi$, where $h_{\mathrm{SM}}$ is the lighter one, $\phi_{-}$, and DM mass is in the region $1598 \mathrm{GeV}<$ $m_{X}<4442 \mathrm{GeV}$.

But $\sigma_{\mathrm{SI}}$ may be not sufficiently suppressed, and consequently the DM direct detection significantly shrinks the viable
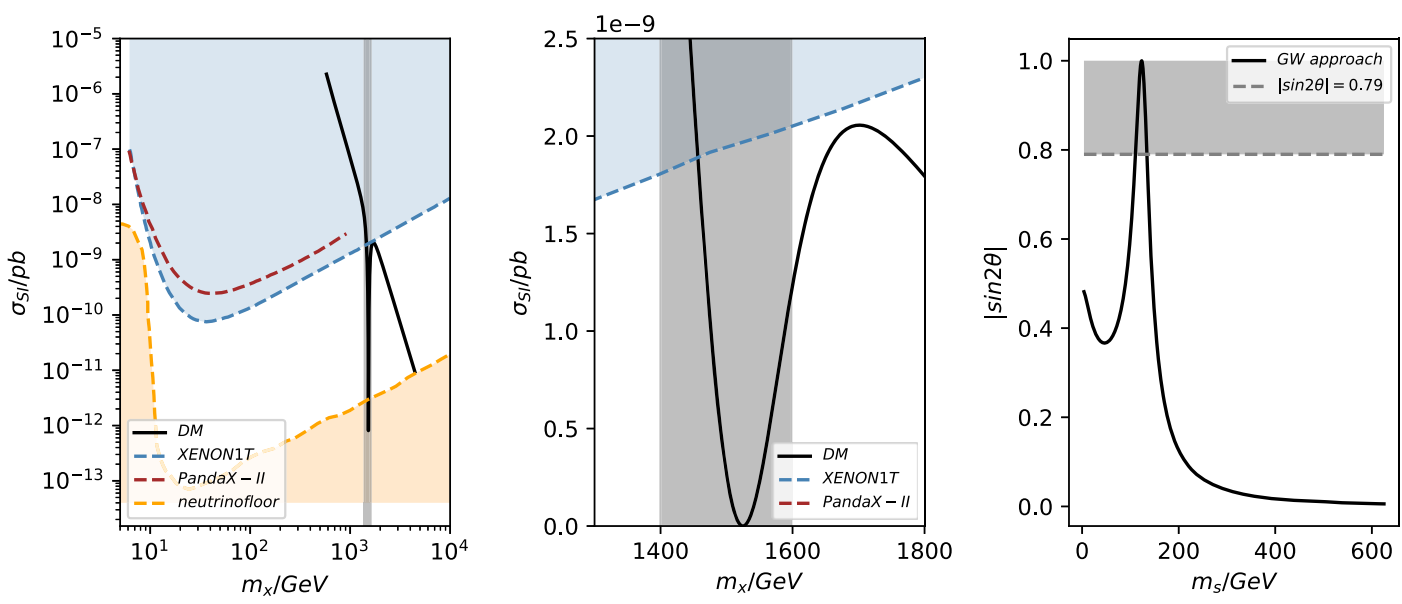

FIG. 2. Left: the surviving parameter space under the DM direct detection bound in the Gildener-Weinberg scenario. Middle: the zoom region showing cancellation. Right: profile of the scalon in the $m_{\mathcal{S}}-|\sin 2 \theta|$ plane. The gray shaded region is excluded by Higgs data. 
regions; see Fig. 2. The relatively light DM region has been excluded except for the narrow trough, which shows subtle cancellation as discussed below Eq. (3.5).

Only the relatively heavy DM mass region $m_{X}>$ $1.56 \mathrm{TeV}$ survives, which indicates $\lambda_{s x}>1.03$ and $m_{\mathcal{S}}>117 \mathrm{GeV}$. The scalon in the Gildener-Weinberg scenario is relatively light and moreover has a larger mixing angle, so it has better prospect at the LHC.

\section{SUPERCOOLING CSI PHASE TRANSITION}

Having studied CSI radiatively breaking at zero temperature, in this section, we go back to the early Universe with high temperature, where CSI is recovered. Of interest, it is found that the transition from the CSI phase to its broken phase is first order, usually characterized by a large supercooling [18-26].

\section{A. Effective potential from finite-temperature correction}

Cosmic PT is based on the finite-temperature effect. When the background fields couple to a bath of plasma, its potential receives temperature-dependent corrections from the thermal fluctuations of the plasma. The leading-order finite-temperature correction takes the form [40]

$V_{T}^{(1)}(\phi, T)=\frac{T^{4}}{2 \pi^{2}}\left(\sum_{a \in \text { boson }} n_{a} J_{B}\left(x_{a}\right)+\sum_{a \in \text { fermion }} n_{a} J_{F}\left(x_{a}\right)\right)$,

with $x_{a}=m_{a}(\phi) / T$. The formalism applies to $\phi$ with multicomponents. Like Eq. (2.5), the index $a$ should run all heavy particles that couple to the backgrounds, e.g., top quark and DM whose masses are given in Eqs. (2.7) and (2.9). When working in the Higgs portal scenario, we only need to include DM and scalon because other particles are massless at the stage of CSIPT. In particular, the absence of Higgs VEV simplifies the masses of scalon and DM to be

$$
m_{s}^{2}=b_{X} s^{2}, \quad m_{X}^{2}=\frac{\lambda_{s x}}{2} s^{2} .
$$

We will use those mass and potentials in following discussion.

In Eq. (4.1), $J_{B}$ and $J_{F}$ are the thermal functions for bosons and fermions, and they, respectively, are given by

$$
J_{B / F}(y)=\int_{0}^{\infty} d x x^{2} \log \left(1 \mp e^{\sqrt{x^{2}+y^{2}}}\right) .
$$

In the $y^{2} \ll 1$ limit, the above integrals admit the hightemperature expansion, up to the quartic terms, taking the forms [40]

$$
\begin{aligned}
& J_{B}(y) \simeq-\frac{\pi^{4}}{24}+\frac{\pi^{2}}{12} y^{2}+\frac{\pi}{6} y^{3}-\frac{1}{32} y^{4} \log \frac{y^{2}}{a_{b}}+\mathcal{O}\left(y^{4}\right) \\
& J_{F}(y) \simeq \frac{7 \pi^{4}}{360}-\frac{\pi^{2}}{24} y^{2}-\frac{1}{32} y^{4} \log \frac{y^{2}}{a_{f}}+\mathcal{O}\left(y^{4}\right),
\end{aligned}
$$

with $\log a_{b} \approx 5.4$ and $\log a_{f} \approx 2.6$. One should be cautious about high-temperature expansion in the PT with a large supercooling, where PT is completed at a very low $T$ and thus $y^{2} \ll 1$ does not hold. But this approximation is still adopted in some of the literature to analyze such a kind of PT, just retaining the quadratic terms. We will come back to this point in Sec. IV D, where we argue how the expansion may still work.

The one-loop effective potential may be insufficient to describe PT. According to the principle that symmetry should be restored at high temperature, the ordinary perturbative expansion in terms of coupling must break down at high temperature [44,45], e.g., around or above the critical temperature $T_{c}$. To improve the expansion so as to make the analysis valid at high $T$, one should sum the highorder diagrams which consist of the quadratically divergent loops on the top of the one-loop self-energy diagram for the spin-0 particles. This procedure yields a thermal correction to their masses,

$$
M_{a}^{2}(\phi, T)=m_{a}^{2}(\phi)+\Pi_{a}(T),
$$

with $\Pi_{a}(T)$ specific to our model given by

$$
\begin{aligned}
\Pi_{h}(T)= & \Pi_{\mathrm{GSB}}=\frac{\lambda}{4} T^{2}+\frac{\lambda_{h s}+\lambda_{h x}}{24} T^{2} \\
& +\frac{3 g^{2}+g^{\prime 2}}{16} T^{2}+\frac{y_{t}^{2}}{4} T^{2}, \\
\Pi_{s}(T)= & \frac{\lambda_{s}}{4} T^{2}+\frac{\lambda_{h s}+\lambda_{s x}}{24} T^{2}, \\
\Pi_{X}(T)= & \frac{\lambda_{x}}{4} T^{2}+\frac{\lambda_{h x}+\lambda_{s x}}{24} T^{2},
\end{aligned}
$$

and also the longitudinal components of the gauge bosons

$\Pi_{W_{L}}(T)=\frac{11}{6} g^{2} T^{2}, \quad \Pi_{Z_{L}}(T) \approx \frac{11}{6}\left(g^{2}+g^{\prime 2}\right) T^{2}$.

We have neglected $\gamma_{L}$, which is not important numerically. Daisy resummation generates the Daisy term in the effective potential $[46,47]$,

$V_{D}(\phi, T)=-\frac{T}{12 \pi} \sum_{a \in \text { boson }} n_{a}\left(\left[m_{a}(\phi)^{2}+\Pi_{a}(T)\right]^{\frac{3}{2}}-m_{a}(\phi)^{3}\right)$,

where $a$ runs over the spin- 0 fields and the longitudinal components of the gauge bosons that appear in Eqs. (4.6) and (4.7) with $n_{W_{L}}=2, g_{Z_{L}}=1$. 


\section{B. Bubble nucleation rate}

According to the work of Coleman and Callan [48], firstorder PT proceeds via the bubble nucleation of the true vacuum. The bubble nucleation rate per volume and per time $\Gamma(T)$, due to thermal fluctuations, is given by [49]

$$
\Gamma \approx A T^{4} e^{-\frac{S_{3}(T)}{T}},
$$

where $A$ is supposed to be at order $1 . S_{3}$ is the $O(3)$ symmetric three-dimensional Euclidean action

$S_{3}(T)=4 \pi \int_{0}^{\infty} R^{2} d R\left[\frac{1}{2}\left(\frac{d \phi}{d R}\right)^{2}+V_{\mathrm{eff}}(\phi, T)\right]$,

with $\quad R=\vec{x}^{2} \quad$ and $\quad V_{\text {eff }}(\phi, T)=V^{(0)}(\phi)+V^{(1)}(\phi)+$ $V_{T}^{(1)}(\phi, T)+V_{D}(\phi, T)$ the total effective potential. $\phi(R)$ is the bounce solution satisfying the Euclidean equation of motion

$$
\frac{d^{2} \phi}{d R^{2}}+\frac{2}{R} \frac{d \phi}{d R}=V_{\mathrm{eff}}^{\prime},
$$

with the boundary conditions $\lim _{R \rightarrow \infty} \phi(R)=0$ (the false vacuum position) and $\left.\frac{d \phi(R)}{d R}\right|_{R \rightarrow 0}=0$. The bounce solution connects the true vacuum and the false vacuum, with phase interface; namely, the bubble wall localized at $R=0$ and $R$ denotes the distance to the wall. The region $R>0(<0)$ is in the symmetric (broken) phase.

Denote $S_{3}(T) / T$ as $S(T)$ hereafter. Finding $S(T)$ or essentially the bounce solution is the basis to discuss PT and also the gravitational wave; however, it heavily relies on the numerical codes, e.g., the PYTHON program CosmoTransition [50]. We will assess the Gildener-Weinberg approach at $T \neq 0$ and Witten's analytical approximation specific to CSIPT.

\section{Multifield: Tunneling along the flat direction versus full tunneling}

In the Gildener-Weinberg approach dealing with radiative symmetry breaking in the multifield space at $T=0$, analysis is done around the valley of the potential, while the calculation of finite-temperature correction is also implemented along the flat direction [see Eq. (B1)], which means that we are assuming that thermal tunneling between the vacua is along this direction. However, a strong quantum correction, present in the case of a larger coupling, may strongly distort the shape of the valley, and therefore we may wonder if the actual tunneling still follows the flat direction. To that end, we study a few example points without considering any phenomenological constraints, and the results are shown in Fig. 3. Two cases of flat direction are presented: one is along $h=s$, while the other one is along $h=s / \sqrt{3}$. From the left panels, one can see that, when $\lambda_{s x}$ becomes sufficiently large, which enhances quantum correction, the tunneling path begins to significantly deviate away from the flat direction. Note that for a given $\lambda_{s x}$ quantum correction leads to a larger deviation for the case with a larger $s$, because the correction mainly comes from the trigger- $s$ coupling. In fact, given that $s \gg h$ holds during tunneling, the multifield problem effectively is reduced to the single-field problem in the sense of calculating $S(T)$.

However, $S(T)$ is not very sensitive to the tunneling path but sensitive to the position of the escaping point. To show this, we calculate $S(T)$ for $\lambda_{s x}=1.2,2.0$, and 3.14, using both the Gildener-Weinberg approach, where tunneling faithfully tracks the flat direction, and the multifield full tunneling, where tunneling is along the actual trajectory, to get

$S(T=10 \mathrm{GeV})=387(335), \quad 139(138), \quad$ and $\quad 50(62)$,

respectively; values in the brackets are for the full tunneling. Analysis on the variation of $S(T)$ with $\lambda_{s x}$ will be given in Sec. IV D. The above examples indicate that the difference between the two ways is mild, typically below $20 \%$. Moreover, it seems that the degree of difference has no simple correlation with the degree of path deviation. In summary, tracking the tree-level flat direction still provides an acceptable approximation to study PT, even facing a relatively strong quantum correction. This conclusion is further supported by a realistic example in Fig. 4, and its first diagram is a comparison between $S(T)$ from two approaches in a wide region of temperature.

\section{Single field: The modified Witten approximation}

If the CSIPT involves only one scalar field, the Witten approximation is usually adopted to estimate $S(T)$ at the very low $T$ region [24], for instance, in the conformal local $B-L$ model [51]. Witten observed that for very low $T$ the field contributing to tunneling extends to $\phi \sim T / \lambda$ with $\lambda$ denoting the coupling between $\phi$ and trigger. ${ }^{6}$ This fact allows one to take high-temperature expansion to derive the tunneling potential merely describing the tunneling process; it is approximated to be

$$
V_{\text {tun }}(\phi, T)=\frac{m_{\mathrm{eff}}^{2}(T)}{2} \phi^{2}+\frac{\lambda_{\mathrm{eff}}(T)}{4} \phi^{4},
$$

where $m_{\mathrm{eff}}^{2}(T)$ is the effective mass in high-temperature expansion and $\lambda_{\text {eff }}(T)$ is negative at low $T$. The vacuum decay of such a potential has been studied in Ref. [52], giving an analytical expression of

\footnotetext{
${ }^{6}$ One may prove this directly from the one-loop effective potential, by finding its zero points, with one at the origin of the metastable vacuum and the other one at the escaping point.
} 
$\alpha=45^{\circ}$
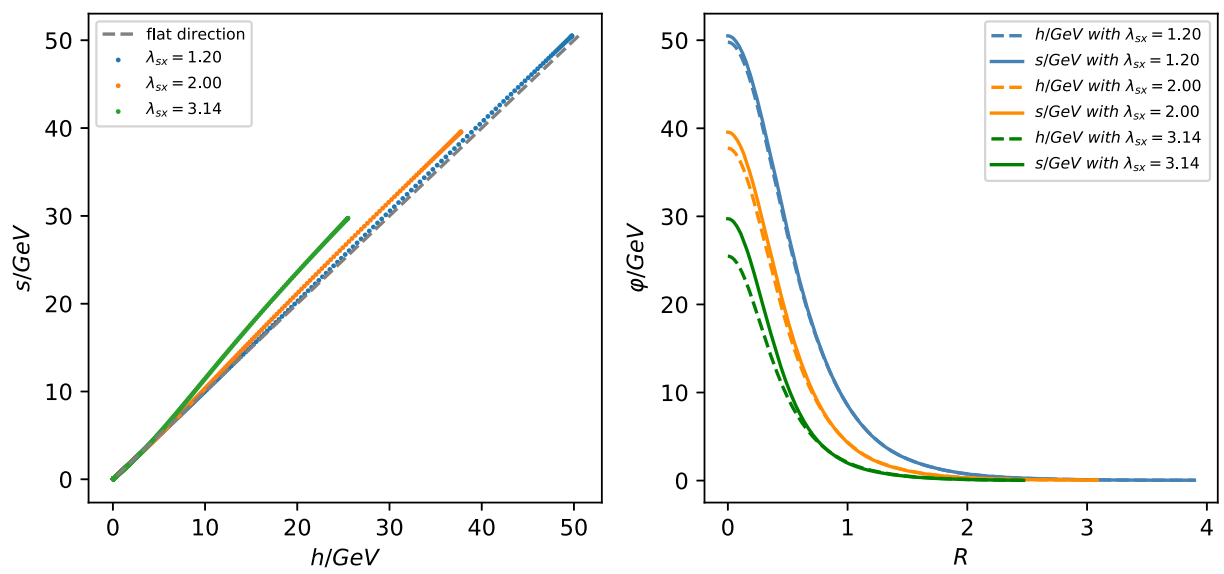

$\alpha=60^{\circ}$
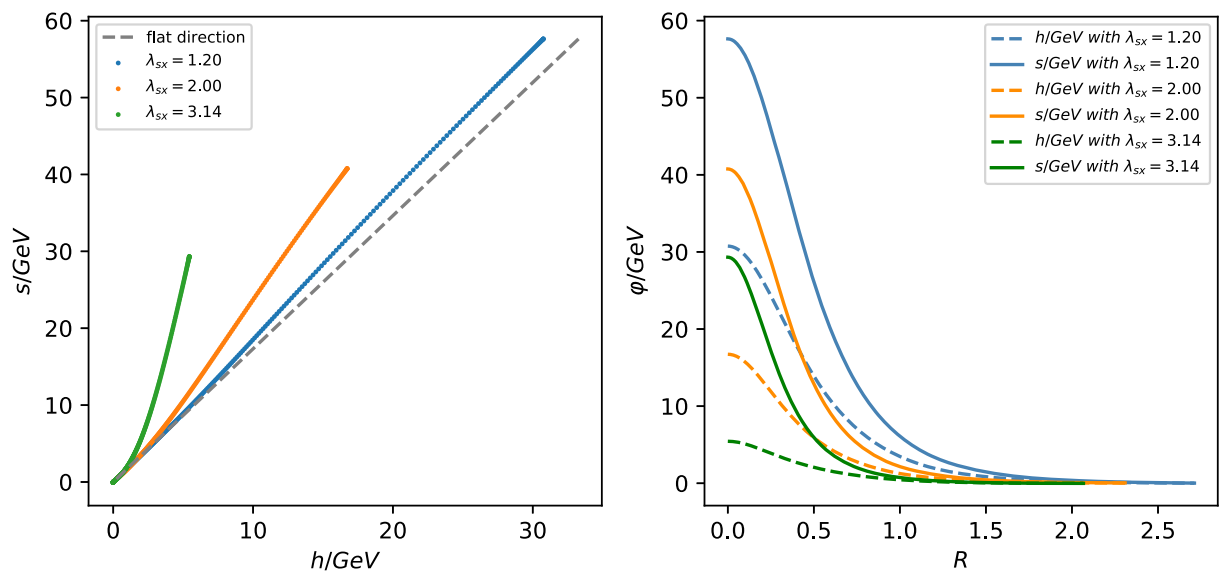

FIG. 3. In this diagram $\alpha$ is the flat direction; all fields values are terminated around the escaping points, after which the particle follow the classical path and thus does not contribute to tunneling. Then, setting $\mathrm{T}=10 \mathrm{GeV}$, we find the solution of tunneling problem with parameter $\lambda_{h}=0.13, \lambda_{h x}=0.001 \lambda_{x}=0.2$ and $v_{h}=246 \mathrm{GeV}$. Other parameter is shown in this diagram or calculated by GildenerWeinberg method.
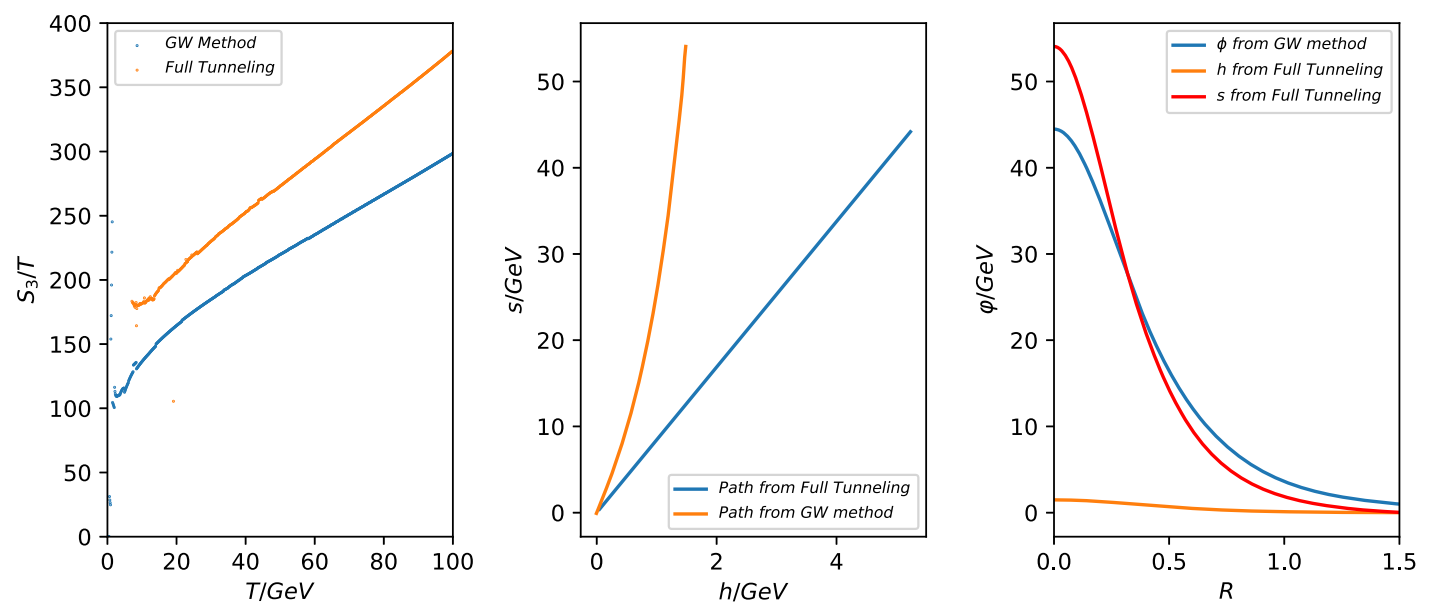

FIG. 4. Left panel: comparison of $S(T)$ between the calculation along the flat direction (top) and along the actual path (bottom). Middle panel: the tunneling path in the $s-h$ space. Right panel: the tunneling path in the $R-\phi$ space. The parameter set is $\lambda_{s x}=1.2$, $\lambda_{h}=0.1273, \lambda_{s}=0.000025$, and $v_{s}=2076 \mathrm{GeV}$. 


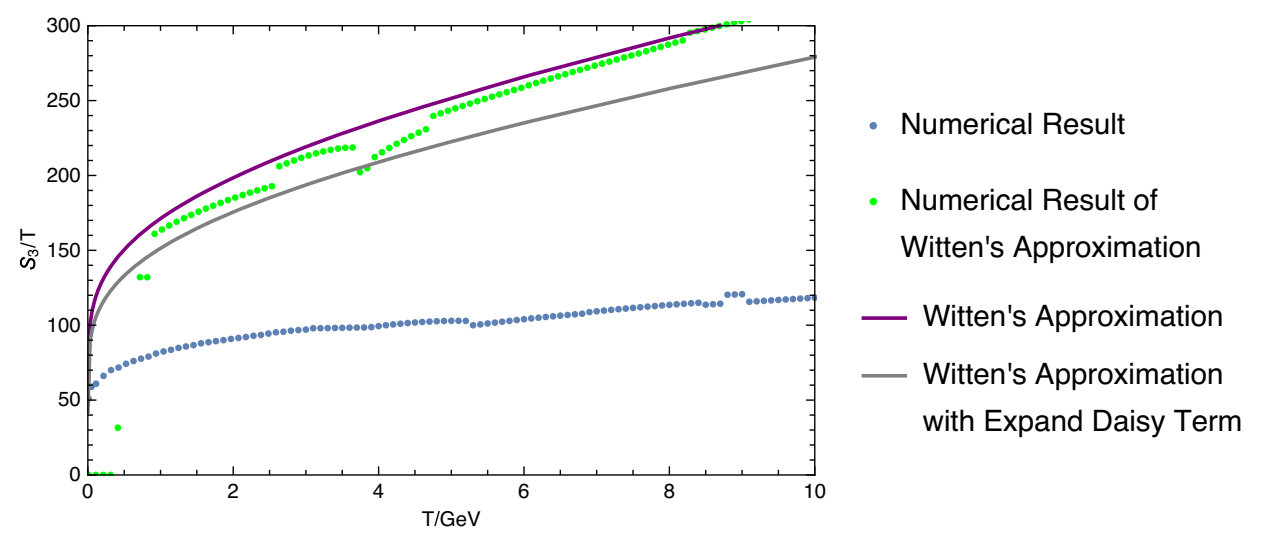

FIG. 5. Various Witten approximations versus the numerical results: the numerical results for the full potential (bottom dotted line) and for the tunneling potential from the original Witten approximation (dotted green line), which indeed is almost the same as its analytical result (top line) and Witten's approximation for the potential after expanding the Daisy term as in Eq. (4.17) (gray solid line).

$$
S(T) \approx-18.897 \frac{m_{\mathrm{eff}}(T)}{T \lambda_{\mathrm{eff}}(T)} .
$$

Nevertheless, the Witten approximation scheme just gives an estimation on $S(T)$ at very low $T$, and it is not good in the sense of precision. Here are two reasons:

(i) First, in the original treatment, only the quadratic term is kept in the high-temperature expansion. In particular, the cubic term $\phi^{3}$, which plays an important role in the shape of the barrier, is simply dropped; on the other hand, keeping this term, one cannot write the tunneling potential in the form of Eq. (4.13). Such over simplification gives rise to a significant deviation to the complete result. If one includes the Daisy term (it is not included in the Witten's paper either), it will exactly cancel that $\phi^{3}$ term but leave the cubic term of the thermally corrected trigger mass.

(ii) Second, the crucial negative quartic coupling is not unique because it is derived by a rough argument rather from the first principle: around the escaping point $\phi \sim T / \lambda$, the logarithmic term in the $\mathrm{CW}$ potential $\log \frac{\phi}{\mu}=\ln \frac{T}{m}+\ln \frac{\lambda \phi}{T} \sim \ln \frac{T}{m}<0$, where $m \simeq$ $\lambda \mu$ is the physical mass of the trigger. The drop of the $\ln \frac{\lambda \phi}{T}$ term is justified in the very small $\phi \ll T$ region, because the quartic term is irrelevant. But obviously, one has some degree of arbitrariness to split $\log \frac{\phi}{\mu}$. Actually, a similar expression can be derived if we keep terms up to the quartic term in the hightemperature expansion. This quartic term and the quartic term in the $\mathrm{CW}$ potential have similar coefficients, and they combine to form

$$
\log \frac{a_{b} T^{2}}{\phi^{2}}+\log \frac{\phi^{2}}{\mu^{2}}=\log \frac{a_{b} T^{2}}{\mu^{2}} .
$$

So, the negative quartic coupling is derived without turning to $\phi \sim T / \lambda$.
In the above discussions, we actually modify the Witten approximation, maintaining the high-temperature expansion (to quartic terms) but giving up the formula (4.14).

To be more specific, we apply the modified Witten approximation to our model in the hidden $\mathrm{CW}$ scenario, only taking into account the DM field $X$. First of all, hightemperature expansion indeed works well. In Fig. 5, the blue dotted line denotes the numerical result of the complete potential, and it coincides well with the line (not plotted explicitly) for the potential in high-temperature expansion. Then, we derive the tunneling potential from the high-temperature expansion,

$$
\begin{aligned}
V_{\text {tun }}(\phi, T)= & \frac{1}{4} \lambda_{s} \phi^{4}+\frac{1}{64 \pi^{2}} m_{X}^{4}(\phi)\left(\log \frac{a_{b} T^{2}}{\mu^{2}}-\frac{3}{2}\right) \\
& +\frac{m_{X}^{2}(\phi) T^{2}}{24}-\frac{M_{X}^{3}(\phi, T) T}{12 \pi} .
\end{aligned}
$$

As mentioned before, the term $M_{X}^{3}(\phi, T) \approx\left(\lambda_{s x} / 2\right)^{3 / 2}\left(\phi^{2}+\right.$ $\left.T^{2} / 12\right)^{3 / 2}$ hampers the direct use of Witten's formula. Hence, we further expand it in terms of $\phi / T$, up to the quadratic term, and then the tunneling potential takes the form of Eq. (4.13) with

$$
\begin{aligned}
& m_{\mathrm{eff}}^{2}(T)=\frac{m_{X}^{2}(T)}{12}-\frac{m_{X}^{2}(T)}{4 \pi} \sqrt{\frac{6 \lambda_{s}+\lambda_{s x}}{24}} \\
& \lambda_{\mathrm{eff}}(T)=\lambda_{s}+\frac{\lambda_{s x}^{2}}{64 \pi^{2}}\left(\log \frac{a_{b} T^{2}}{\mu^{2}}-\frac{3}{2}\right)
\end{aligned}
$$

To check if this approximation works well, we compare the resulting $S(T)$ by Witten's formula with the complete numerical results, to find that it is a poor approximation; see Fig. 5. This inaccuracy is owing to the fact that the expansion $\phi / T \sim \mathcal{O}(1)$ is multiplied by a large factor $12 \lambda_{s x} \gg 1$. Therefore, we draw the conclusion that Witten's formula does not give a precise estimation on $S(T)$. 


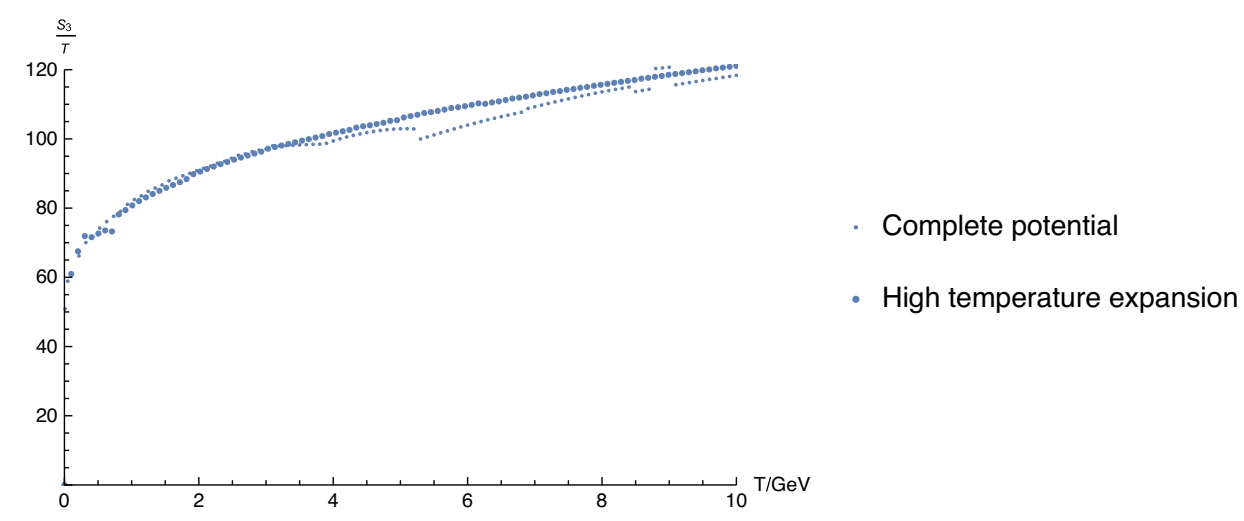

FIG. 6. The difference between the generalized Witten argument and complete potential is very small; at very low temperature, they nearly overlap with each other. So, gravitational signal from them would also overlap.

\section{Multilfield: Possible generalized Witten's argument}

Maybe the essence of the Witten argument is not the poor formula for estimating $S(T)$ but the observation that hightemperature expansion is a good approximation to encode the tunneling dynamics far below the critical temperature. More concretely, the barrier, in particular, the escaping point, just extends over the small field region, and thus the quantum tunneling path merely tracks small fields, whereas the large fields, where the ground state is located, are irrelevant. This fact, along with the CSI, confers the legitimacy of the high-temperature expansion at very low temperature, for example, Fig. 6.

The original argument is for the single field, and we conjecture that it may also apply to the multifield case. But it is difficult to prove it explicitly since, unlike the onedimensional case, the escaping points now are located in a hypersurface in the $n$-dimensional field space and we are incapable of pinning down the exact point at which the tunneling ends. Moreover, the scale of fields varies widely on the escaping hypersurface, which renders the failure of the simple conclusion that the tunneling process just involves fields extending to $T / \lambda$. But we conjecture it is true. A support is from the left panel of Fig. 7, where the contours are the equipotential lines of our model, and the thick black line with zero potential energy is the escaping line. Its interaction with the straight line, the tunneling path, is the actual escaping point. Thus, as our conjecture, the tunneling is through the small field region and ends at the small field, and then Witten's argument is supposed to hold.

Even if the above generalization is true, to compute $S(T)$, we still have to rely on the numerical codes. Our discussions help to clarify what is the correct way of using the high-temperature expansion in CSIPT; some authors merely keep the quadratic terms of $T$, but it does not give good enough numerical results; more details can be found in Appendix. B. To have a sufficiently good result, we need to expand the finite-temperature potential to the quartic terms. In the right panel of Fig. 7, we show the quality of high-temperature expansion in this scheme, measured by $\delta S(T) / S(T)$ : the difference between the $S(T)$ calculated

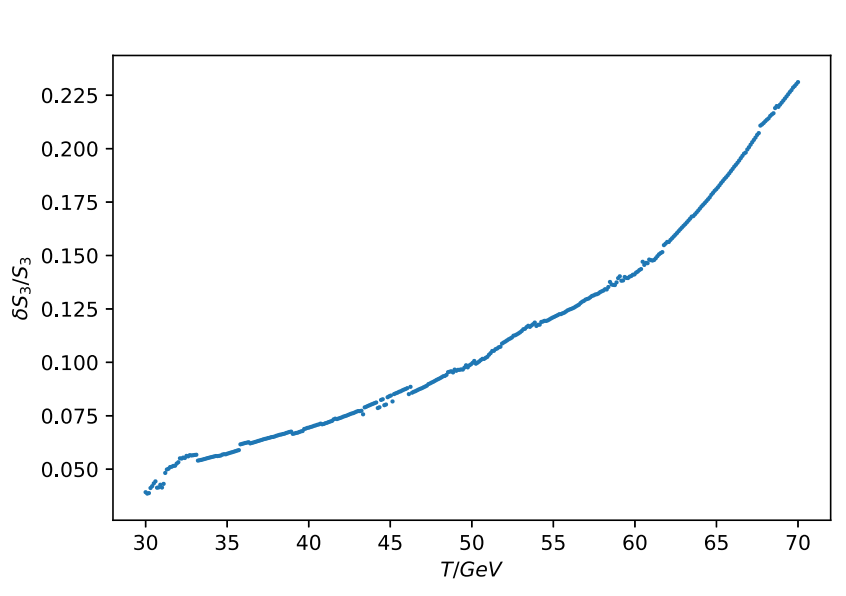

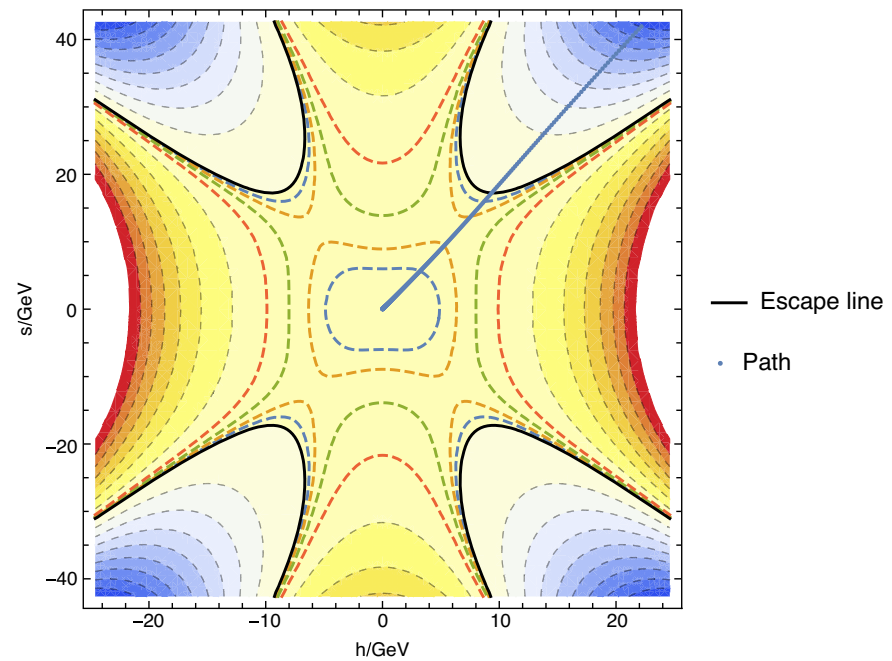

FIG. 7. Left panel: contours of equipotential lines of the model and the tunneling path (straight line). Right panel: the relative error of $S(T)$ for high-temperature expansion. 
using the complete potential and the expanded one, normalized by the complete result. We can see that the quality is steadily improved as temperature decreases, contrast to the behavior of normal high-temperature expansion.

\section{CSIPT in a hot bath or in the vacuum?}

As a consequence of very strong supercooling CSIPT, the early Universe experienced a very short stage of a vacuum-dominated era and thus a short period of little inflation. CSIPT may be completed during this epoch rather than the usual radiation-dominated (RD) era, and then we should reconsider the condition of CSIPT completion, which was recently stressed by the authors of Ref. [26,53]. This is not very new, and the discussion is reminiscent of the old inflation idea proposed by Guth [54], but here the little inflation will be ended by thermal instead of quantum tunneling.

\section{Little inflation}

In the scenario of supercooling PT, the Universe was confined in the false vacuum until the PT completion temperature $T_{*}$, which lies much below the critical temperature $T_{c}$. In this vacuum, the nonvanishing vacuum energy density $\rho_{0}$ is nearly a constant (we will come back to this point soon later), so it may begin to exceed the radiation energy density $\rho_{r}(T)=\frac{\pi^{2}}{30} g_{*} T^{4}$ at some lower temperature $T_{V} \simeq\left(30 g_{*} \rho_{0} / \pi^{2}\right)^{1 / 4}$, where $g_{*} \sim 100$ is the relativistic degrees of freedom in the false vacuum plasma.

$\rho_{0}$ is determined by the potential energy of the false vacuum. The effective scalar potential has $T$ dependence, and therefore in principle $\rho_{0}$ also depends on $T$. However, since we are interested in the region near $T_{*}$, which is low due to supercooling, the finite-temperature effect becomes fairly weak. Roughly speaking, this effect merely reshapes the potential near the origin (the small field region), maintaining the local minimum; it does not significantly change the ground state (the relatively large field region). So, it is a good approximation to calculate the vacuum energy from the effective energy at $T=0^{7}$ :

$$
\rho_{0}=V_{0}^{(1)}\left(0, T_{n}\right)-V_{0}^{(1)}\left(\langle\phi\rangle, T_{n}\right)=\frac{1}{2} B\langle\phi\rangle^{4} .
$$

It is for the Gildener-Weinberg scenario, and a similar result can be derived in the Higgs portal scenario. As a result of CSI, its scale is mainly determined by the position of the ground state. Then, $T_{V}$ is estimated to be

\footnotetext{
${ }^{7}$ The true vacuum energy should be fine-tuned to be zero by adding a constant to the potential, which is the usual cosmological constant problem. This constant is not dimensionless, thus explicitly breaking CSI. We do not have an approach to reconcile CSI with it in this paper.
}

$$
T_{V} \simeq\left(\frac{15}{\pi^{2}} \frac{B}{g_{*}}\right)^{\frac{1}{4}} v_{\phi}
$$

The prefactor is about 0.1 for a normal loop function $B \sim 10^{-2}$, and moreover we are considering $v_{\phi}$ at the $\mathrm{TeV}$ scale, so typically $T_{V} \sim 100 \mathrm{GeV}$.

After the Universe energy density is dominated by vacuum energy, the size of the Universe grows exponentially by means of inflation,

$$
a(t)=a_{V} e^{H_{V}\left(t-t_{V}\right)},
$$

where $H_{V} \approx \rho_{0}^{1 / 2} /\left(\sqrt{3} M_{\mathrm{Pl}}\right)$ is the Hubble parameter during the vacuum-dominated era; $t_{V}$ and $a_{V}$ are the time and scale factor at $T_{V}$, respectively. As in the inflation, we denote the Hubble times of $1 / H_{V}$ as the $e$-folding number $N$ : $t_{N}-t_{V}=N / H_{V}$. Here, we consider the inflation with a smaller $N$ of a few, thus the little inflation. The temperature of the radiation drops exponentially, $T(t)=T_{V} e^{-H_{V}\left(t-t_{V}\right)}$, and for $T_{V} \sim 100 \mathrm{GeV}$, the Universe cools down to the sub$\mathrm{GeV}$ after about $N \sim 6$ Hubble times. If the CSIPT fails to complete before it, the QCD chiral PT will terminate inflation around this temperature [55]. In this paper, we focus on the case in which CSIPT is capable of ending inflation. ${ }^{8}$ In the following, we investigate the condition for a successful CSIPT.

\section{Condition for CSIPT completion}

Despite of the difficulty of getting an analytical expression for $\Gamma(T)$, practically, it is sufficient to be aware of a fact like the following; in general, $\Gamma(T)$ monotonically decreases with $T$ since $S(T)$ increases with $T$. Therefore, the integration involving $\Gamma(T)$ is supposed to be dominated by the lower bound (in some sense, insensitive to UV). Then, one has the useful approximation

$$
\begin{aligned}
\int_{T}^{T_{c}} \Gamma\left(T^{\prime}\right) T^{\prime n-4} d T^{\prime} & \approx \int_{T}^{T_{c}} A e^{-\beta_{0} T^{\prime}} T^{\prime n} d T^{\prime} \\
& \approx A \beta_{0}^{-n-1} e^{-S\left(T_{0}\right)} \Gamma\left(n+1, \beta_{0} T\right),
\end{aligned}
$$

where we have expanded $S(T)$ around some temperature $T_{0}: S(T)=S\left(T_{0}\right)+\beta_{0}\left(T-T_{0}\right)+\ldots$, retaining only the linear term. Note that $\beta_{0} \equiv d S(T) /\left.d T\right|_{T_{0}}>0$. This treatment works very well for $T$ sufficiently close to $T_{0}$ and also $T_{c} \gg T \sim T_{0}$. As a matter of fact, we will study $S\left(T_{0}\right) \sim \mathcal{O}(10)$, so it always works.

When does the bubbles of true vacuum overwhelmingly occupy the space of false vacuum? We label this temperature (time) as $T_{n}\left(t_{n}\right)$, known as the bubble nucleation temperature (time). In the RD epoch, the criterion is that at $T_{n}\left(t_{n}\right)$ a single bubble is nucleated within one Hubble horizon volume,

\footnotetext{
${ }^{8}$ Baryon asymmetry may be an issue if $N$ is very large.
} 


$$
\begin{aligned}
N_{n} & =\int_{t_{c}}^{t_{n}} d t \frac{\Gamma(t)}{H(t)^{3}} \\
& =A \int_{T_{n}}^{T_{c}} \frac{d T}{T^{5}}\left(3 M_{\mathrm{Pl}}^{2}\right)^{2}\left(\frac{30}{\pi^{2} g_{*}}\right)^{2} e^{-S(T)} \sim 1
\end{aligned}
$$

To perform the integration over temperature, which is more convenient in PT, we have utilized the expansion rate in the RD epoch, $H(T)^{2}=\frac{\pi^{2} g_{*}}{30} T^{4} /\left(3 M_{\mathrm{Pl}}^{2}\right)$ with $M_{\mathrm{Pl}}=$ $2.43 \times 10^{18} \mathrm{GeV}$ and also the time-temperature relation

$$
d t / d T=-1 /(H T)
$$

It holds for the Universe evolving adiabatically, true both in the radiation- and vacuum-dominated eras considered in this paper. Then, following Eq. (4.21), the condition (4.22) is translated to the well-known equation

$$
\begin{aligned}
S\left(T_{n}\right) \simeq & 2 \log \left(3 M_{\mathrm{Pl}}^{2} / T_{n}^{2}\right)+2 \log \frac{15}{\sqrt{6} \pi^{2} g_{*}} \\
& +\log f_{R}(x) \sim 140,
\end{aligned}
$$

where $f_{R}(x)=-6+2 x-x^{2}+x^{3}+x^{4} e^{x} \operatorname{Ei}(x)$ with $x \equiv$ $\beta_{n} T_{n} \sim \mathcal{O}(1)$ in our samples of numerical calculations.

But $S\left(T_{n}\right) \sim 140$ significantly overestimates the required value of $S\left(T_{n}\right)$ in the vacuum-dominated epoch. Estimates Eq. (4.22) in this epoch where $H=H_{V}$, the condition Eq. (4.24) turns out to be

$$
S\left(T_{n}\right) \simeq 2 \log \left(3 M_{\mathrm{Pl}}^{2} / T_{n}^{2}\right)+2 \log T_{n}^{4} / \rho_{0}+\log f_{V}(x),
$$

with $f_{V}(x)=\left(6+6 x+3 x^{2}+x^{3}\right) / x^{4}$. It is similar to the usual nucleation condition (4.24), but the term $2 \log \rho_{0}$, originating in vacuum dominance, brings a significant numerical difference; now, typically $S\left(T_{n}\right) \sim 70$. The concrete value of $\beta_{n}$, found to be approximately $\mathrm{GeV}^{-1}$ for a wide region of temperature in our model [which indicates that $S(T)$ is almost linear in $T$ ], is almost irrelevant in calculating $S\left(T_{n}\right)$.

The above bubble nucleation condition does not reflect the progress of PT, so one may develop a more apparent criterion via $P(t)$, the probability of a space point staying in the false vacuum [56]. The criterion $P(t) \lesssim 70 \%$ is usually used to fulfill percolation in the three-dimensional Euclidean space. ${ }^{9} P(t)=e^{-I(t)}$ with $I(t)$ the expected volume of true-vacuum bubbles per unit volume of space at time $t$ [57], explicitly

\footnotetext{
${ }^{9}$ Successful bubble percolation is required to make the space homogeneous; namely, the bubbles do not form finite clusters. It is a more strict condition for PT completion.
}

$$
I(t)=\int_{t_{c}}^{t} d t^{\prime} \Gamma\left(t^{\prime}\right) a\left(t^{\prime}\right)^{3} V\left(t, t^{\prime}\right), \quad V\left(t, t^{\prime}\right)=\frac{4 \pi}{3} r\left(t, t^{\prime}\right)^{3},
$$

with

$$
r\left(t, t^{\prime}\right)=\int_{t^{\prime}}^{t} v_{w}\left(t^{\prime \prime}\right) \frac{d t^{\prime \prime}}{a\left(t^{\prime \prime}\right)}
$$

being the comoving radius of the bubble nucleated at $t^{\prime}$ expanding with a velocity $v_{w}$ until $t$. However, $P(T)$ alone may be insufficient to judge whether the phase transition is completed: In the vacuum dominated era, the $P(T)$ can be arbitrarily small but PT is never completed because of the inflation of the false vacuum [58].

In such a case, a better condition for successful PT completion is obtained by finding the time $T_{e}$ since which the physical volume of the false vacuum $\mathcal{V}_{f}(T)=$ $a(T)^{3} P(T)$ commences shrinking [56]. It leads to the following condition:

$\frac{1}{\mathcal{V}_{f}(t)} \frac{d \mathcal{V}_{f}(t)}{d t}=3 H(t)-\frac{d I(t)}{d t}=H(T)\left(3+T \frac{d I(T)}{d T}\right) \leq 0$

For further analysis, we should pursue an approximation to $I(t)$. Because the bubble is very energetic in the very strong $\mathrm{PT}$, it is safe to take $v_{w}(t) \approx 1$. Then, utilizing Eq. (4.21) and working in the vacuum-dominated era, one can get ${ }^{10}$

$$
I(T) \approx A \frac{8 \pi}{\beta_{0}^{4} H_{V}^{4}} \exp \left[-S\left(T_{0}\right)-\beta_{0}\left(T-T_{0}\right)\right]
$$

Now, saturating the equality (4.28) and taking advantage of Eq. (4.29) yields the equation $3-T_{e} \beta_{e} I\left(T_{e}\right)=0$, or more concretely the PT completion condition

$$
\begin{aligned}
3-\frac{8 \pi e^{-S\left(T_{e}\right)} T_{e}}{\beta_{e}^{3} H_{V}^{4}}= & 0 \Rightarrow S\left(T_{e}\right)=2 \log \frac{3 M_{\mathrm{Pl}}^{2}}{T_{e}^{2}} \\
& +2 \log \frac{T_{e}^{4}}{\rho_{0}}+\log \frac{8 \pi}{3 x^{3}} .
\end{aligned}
$$

It is almost identical to Eq. (4.25) except for the last term that is subdominant; actually, the difference is just a few for a widely changing $x$. Therefore, the difference is not sizable no matter which criteria are used to measure the completion of PT.

The real implication of the latter criterion is that it forces $\mathcal{V}_{f}\left(T_{e}\right)$ to reach a maximum at $T_{e}$, and thus the second term of its Taylor expansion

\footnotetext{
${ }^{10}$ In the RD era, $I_{R D}(T) \approx \frac{\pi}{18 H_{R}(T)^{4}} \Gamma(T) F\left(\beta_{0} T\right)$ with $F(x)=$ $\left(x^{3}+12 x^{2}+36 x+24\right) x e^{x} \operatorname{Ei}(-x)+x^{3}+11 x^{2}+26 x+6>0$.
} 
TABLE I. Benchmark points in the Gildener-Weinberg scenario.

\begin{tabular}{|c|c|c|c|c|c|c|c|c|c|c|}
\hline & $v_{s} / \mathrm{GeV}$ & $\lambda$ & $\lambda_{s}$ & $\lambda_{x}$ & $\lambda_{h x}$ & $\lambda_{s x}$ & $\alpha$ & $\tilde{\beta}$ & $T_{n} / \mathrm{GeV}$ & $T_{*} / \mathrm{GeV}$ \\
\hline A & 2400 & 0.1277 & 0.000014 & 0.2 & $10^{-3}$ & 1.44 & $3.9 * 10^{8}$ & 11.3 & 1.01 & 616 \\
\hline B & 2449 & 0.1278 & 0.000013 & 0.2 & $10^{-3}$ & 1.50 & $6.6 * 10^{7}$ & 9.84 & 1.65 & 646 \\
\hline $\mathrm{C}$ & 2683 & 0.1280 & 0.000009 & 0.2 & $10^{-3}$ & 1.80 & 59805 & 14.36 & 11.40 & 796 \\
\hline $\mathrm{D}$ & 2828 & 0.1281 & 0.000007 & 0.2 & $10^{-3}$ & 2.00 & 2301 & 17.16 & 28.60 & 896 \\
\hline $\mathrm{E}$ & 2966 & 0.1282 & 0.000006 & 0.2 & $10^{-3}$ & 2.20 & 0.37 & 94.73 & 278.63 & 996 \\
\hline $\mathrm{F}$ & 3535 & 0.1285 & 0.000003 & 0.2 & $10^{-3}$ & 3.14 & 0.004 & 198.07 & 750.85 & 1475 \\
\hline $\mathrm{G}$ & 2449 & 0.1278 & 0.000013 & 1.2 & $10^{-3}$ & 1.50 & 370801 & 10.00 & 6.02 & 720 \\
\hline
\end{tabular}

TABLE II. Benchmark points in the Higgs portal scenario.

\begin{tabular}{|c|c|c|c|c|c|c|c|c|c|}
\hline & $v_{s} / \mathrm{GeV}$ & $\lambda$ & $\lambda_{s}$ & $\lambda_{h s}$ & $\lambda_{s x}$ & $\alpha_{n}$ & $\tilde{\beta}$ & $T_{n} / \mathrm{GeV}$ & $T_{*} / \mathrm{GeV}$ \\
\hline $\mathrm{a}$ & 2245 & 0.1304 & -0.00110 & -0.00286 & 1.38 & $3.0 * 10^{8}$ & 10.72 & 1.05 & 576 \\
\hline b & 2449 & 0.1299 & -0.00119 & -0.00262 & 1.50 & $1.4 * 10^{7}$ & 14.75 & 2.43 & 688 \\
\hline $\mathrm{c}$ & 2683 & 0.1294 & -0.00142 & -0.00218 & 1.80 & 16213 & 16.59 & 15.80 & 829 \\
\hline d & 2828 & 0.1293 & -0.00158 & -0.00196 & 2.00 & 0.78 & 82.49 & 28.60 & 923 \\
\hline e & 2966 & 0.1292 & -0.00174 & -0.00178 & 2.20 & 0.30 & 99.90 & 293.83 & 1018 \\
\hline f & 3535 & 0.1291 & -0.00249 & -0.00124 & 3.14 & 0.003 & 203.46 & 753.56 & 1473 \\
\hline
\end{tabular}

$$
\begin{aligned}
\mathcal{V}_{f}(T)= & \mathcal{V}_{f}\left(T_{e}\right) \\
& \times\left(1+\left.\frac{\left(T-T_{e}\right)^{2}}{2}\left(3 / T^{2}-\frac{d^{2} I}{d T^{2}}\right)\right|_{T=T_{e}}+\cdots\right)
\end{aligned}
$$

should have a negative coefficient at $T_{e}$. It contains two competitive pieces. One is from the curvature of $P(T)$, generating the native piece $-d^{2} I /\left.d T^{2}\right|_{T=T_{e}}=-\beta_{e}^{2} I\left(T_{e}\right)=$ $-3 \beta_{e} / T_{e}<0$. The other one is from volume expansion $a^{3}$, generating the positive piece $3 / T_{e}^{2}$. The two pieces add up to a negative coefficient imposing a lower bound on the PT completion temperature, $T_{e}>3 / \beta_{e}$. It is more convenient to rewrite the condition in terms of $\tilde{\beta}$ that will be defined in Eq. (5.2):

$$
T_{e} \beta_{e}=\tilde{\beta}>3 .
$$

By contrast, if PT completes in the RD era, there is no such kind of bound because $\left.\left(3 / T^{2}-d I^{2} / d T^{2}\right)\right|_{T_{e}}=3 / T_{e}^{2} \mathcal{F}(x)$ with $\mathcal{F}(x)$ definitely negative.

\section{Numerical results}

The above discussions did not offer a way to judge the period in which CSIPT happened, and here is our procedure. First, we calculate $S(T)$ and next assume the RD era to determine $T_{n}$ via $S(T) \simeq 140$. If, indeed, the ratio $\alpha_{n} \equiv \rho_{0} / \rho_{r}(T)$ at $T=T_{n}$ is smaller than 1 , then the assumption is justified. Otherwise, CSIPT should be completed in the vacuum-dominance era, and finally we take the criterion $S(T) \simeq 70$ to determine $T_{n}$. This is a simplified procedure, and we refer to Ref. [26] for a more accurate treatment using iteration. In general, their difference is not significant except for the subtle case where $\alpha_{n}$ is close to 1, and hence the era has comparable radiation and vacuum energy density. As a consequence, either criterion works well. We will go back to this point in a later concrete example.

Now, we present the numerical results of CSIPT. We choose a few benchmarks points, which satisfy all the phenomenological constraints and requirements from radiative CSISB and DM discussed before; the condition (4.32) is also imposed. Then, only one free parameter $\lambda_{s x}$ is left except for the irrelevant (to those phenomenologies) ones $\lambda_{x}$ and $\lambda_{h x}$. In Table I, we show the benchmarks points in the Gildener-Weinberg scenario. One can see that $T_{n}$ increases with $\lambda_{s x}$, which is traced back to the decreasing $S(T)$, explicit in Eq. (4.12); in simple terms, the larger quantum (also thermal) correction benefits thermal tunneling. This behavior is explained by the narrower of the barrier, i.e., the shorter escaping path, ${ }^{11}$ with the increasing $\lambda_{s x}$; one can find its evidence in Fig. 3. Note that to make $T_{n}$ lie above the QCD chiral symmetry breaking scale $\lambda_{s x}$ should be sufficiently large, for instance, $\lambda_{s x} \gtrsim 1.44$ in the GildenerWeinberg scenario.

The observed $T_{n}-\lambda_{s x}$ behavior has immediate implications to CSIPT thus gravitational wave, and we can clearly see this from the table. Among the eight benchmarks, A, B, C, D, and G have a relatively small $\lambda_{s x} \lesssim 2.0$,

\footnotetext{
${ }^{11}$ Although not shown here, we find that at the same time the barrier becomes shallower, which brings an opposite effect to $S(T)$, but it is supposed to be subdominant to the former effect.
} 
and the corresponding CSIPTs were completed in the vacuum dominated period. They give a very large $\alpha_{n}$, characterizing strong supercooling. By contrast, CSIPTs of $\mathrm{E}$ and $\mathrm{F}$, which have a relatively larger $\lambda_{s x} \gtrsim 2.2$, were completed in the RD era and give a suppressed $\alpha_{n}$. Therefore, the heavier DM region may be characterized by less vacuum energy release. This is not good news since the heavier DM region is just the region which tends to go beyond the sensitivity of DM direct detection experiments.

We also display the benchmarks for the Higgs portal scenario in Table II, to find that the two scenarios share a fairly similar feature of CSIPT, provided that the values of $\lambda_{s x}$ are close. It is not surprising since CSIPTs in both scenarios are dominated by the singlet scalar, whose quantum corrections dominantly come from the DM field.

\section{ABUNDANT GRAVITATIONAL WAVE (GW) FROM CSIPT}

In the last section, we have shown that CSIPT, due to the vanishing quadratic term of the scalon, is first order and moreover characterized by significant supercooling for the not very heavy trigger. So, the bubble collisions near the end of CSIPT stimulate abundant emission of GW, which may be hunted by eLISA, Tianqin, etc. From the DM direct detection bounds shown in Figs. 1 and 2, it is seen that the multi-TeV DM region is buried underneath the neutrino floor, and consequently it cannot be probed by the DM direct detection experiments. Fortunately, the GW signal opens a window to probe this region.

In estimating the GW spectra, there are two critical parameters which characterize first-order PT, namely, the $\alpha$ and $\tilde{\beta}$ parameters defined as

$\left.\alpha \equiv \frac{\Delta \epsilon}{\rho_{r}}\right|_{T=T_{n}}, \quad \Delta \epsilon=\rho_{0}+T \frac{d}{d T}\left[V_{\mathrm{eff}}\left(\phi_{0}, T\right)-V_{\mathrm{eff}}(0, T)\right]$,

$$
\tilde{\beta} \equiv-\left.\frac{1}{H} \frac{d S(t)}{d t}\right|_{t=t_{n}}=\left.T_{n} \frac{d S(T)}{d T}\right|_{T=T_{n}} .
$$

$\alpha$ denotes the latent heat release $\Delta \epsilon$ normalized by the energy density of radiation during PT. It receives two contributions, but in the strong supercooling PT, it is obviously dominated by $\rho_{0}$, the vacuum energy difference defined in Eq. (4.18), while $\tilde{\beta}^{-1} \sim \tau_{P T} / \tau_{H}$ denotes the time scale of PT duration, normalized by the Hubble timescale $\tau_{H} \sim 1 / H$ at $t_{n}$. The GW amplitude is enhanced by the larger $\alpha$ and $\tilde{\beta}^{-1}$. Their values have been listed in the previous tables.

One may obtain an overall picture about $\tilde{\beta}^{-1}$. The typical behavior of $S(T)$ is plotted in Fig. 5, which leads us to the observation: In the relatively high temperature region $S(T)$ is almost linear in $T$, with a slope $\sim \mathcal{O}(0.5) \mathrm{GeV}^{-1}$, and the slope just slowly increases as $T$ decreases; however, the slope sharply increases when $T$ drops below certain temperature. Thereby, if CSIPT is completed at a higher $T_{n}$, one has $\tilde{\beta} \sim \mathcal{O}(0.5) T_{n} / \mathrm{GeV}$; otherwise, it may be enhanced by orders of magnitude. This observation roughly explains the pattern of $\tilde{\beta}$ in Tables I and II.

\section{A. GW sources}

According to the present understanding of the GW emission during PT proceeding via the thermal bubble nucleation, there are three sources after bubble collision at $T_{n}$ :

(i) Bubble collision: Before the bubble wall reaching the terminal velocity, almost all of the vacuum energy (or latent heat) will be transformed into the kinetic energy of the bubble wall. If the bubble wall is expanding in the vacuum, it runs away, that is to say, it keeps accelerating until bubble collision. Even expanding in a plasma, the bubble was still believed to run away in the strongly supercooling PT with

$$
\begin{aligned}
\alpha>\alpha_{\infty} & \equiv \frac{\Delta P_{\mathrm{LO}}}{\rho_{R}} \approx \frac{30}{24 \pi^{2}} \frac{\sum_{a} c_{a} \Delta m_{a}^{2}\left(\phi_{n}\right)}{g_{*} T_{n}^{2}} \\
& \sim 10^{-2}\left(\frac{\phi_{n}}{T_{n}}\right)^{2} .
\end{aligned}
$$

Nevertheless, recently, it was found that the friction on the wall at the next-to leading order is proportional to the Lorentz factor of the wall, $\Delta P_{\mathrm{NLO}} \propto \gamma$ [59]. It is able to balance the wall when $\gamma \rightarrow \gamma_{\text {eq }}$, thus stopping runaway. Then, the energy stored in the bubble is still negligible (namely, the fraction of latent heat transferred to bubble collision $\kappa_{\mathrm{col}} \ll 1$ ), provided that $\alpha$ does not become extremely large [26], far larger than the $\alpha$ considered in this paper. So, the GW source as usual is from the bulk motion of the plasma.

(ii) Sound wave: The first bulk motion is the sound wave propagating in the plasma after percolation happens. The fraction of latent heat that goes into the fluid motion is estimated to be [60]

$$
\kappa_{s w} \approx \alpha(0.73+0.083 \sqrt{\alpha}+\alpha)^{-1} \stackrel{\alpha \gg 1}{\longrightarrow} 1 .
$$

The GW peak frequency at $T_{n}$ is not well understood and is $f_{s w, *}=2(8 \pi)^{1 / 3} /\left[\sqrt{3}\left(v_{w}-c_{s}\right) R_{*}\right]$ with $R_{*}$ the average bubble separation at collision and the speed of sound in the plasma $c_{s}=1 / \sqrt{3}$. In the exponential approximation of $S(T)$, it is related to the typical timescale of PT: $R_{*}=(8 \pi)^{1 / 3} v_{w} / \beta_{n}$. Redshifting to today, the observed peak is $f_{s w}=$ $f_{s w, *} a_{0} / a\left(T_{n}\right)$ and parametrized as $[26,61]$ 
TABLE III. A subtle case of CSIPT completion criterion.

\begin{tabular}{|c|c|c|c|c|c|c|c|c|c|c|}
\hline & $v_{s} / \mathrm{GeV}$ & $\lambda$ & $\lambda_{s}$ & $\lambda_{x}$ & $\lambda_{h x}$ & $\lambda_{s x}$ & $\alpha$ & $\tilde{\beta}$ & $T_{n} / \mathrm{GeV}$ & $T_{*} / \mathrm{GeV}$ \\
\hline Radiation & 2828 & 0.1281 & 0.000007 & 0.2 & $10^{-3}$ & 2.00 & 1.04 & 77.76 & 196.11 & 896 \\
\hline Mixing & 2828 & 0.1281 & 0.000007 & 0.2 & $10^{-3}$ & 2.00 & 4.10 & 56.42 & 139.15 & 896 \\
\hline Vacuum & 2828 & 0.1281 & 0.000007 & 0.2 & $10^{-3}$ & 2.00 & 2301 & 17.16 & 28.60 & 896 \\
\hline
\end{tabular}

$$
\begin{aligned}
f_{s w}= & 1.65 \times 10^{-5}\left(\frac{T_{n}}{100 \mathrm{GeV}}\right)\left(\frac{g_{*}}{100}\right)^{\frac{1}{6}} \\
& \times \frac{3.4}{\left(v_{w}-c_{s}\right) H_{*} R_{*}} \mathrm{~Hz} \\
= & 2.75 \times 10^{-5} \frac{\tilde{\beta}}{v_{w}}\left(\frac{T_{n}}{100 \mathrm{GeV}}\right)\left(\frac{g_{*}}{100}\right)^{\frac{1}{6}} \mathrm{~Hz}
\end{aligned}
$$

The GW spectrum of the sound wave is $[26,62]$

$$
\begin{aligned}
h^{2} \Omega_{s w}(f)= & 6.35 \times 10^{-6}\left(H_{*} R_{*}\right)\left(H_{*} \tau_{s w}\right) \\
& \times\left(\frac{\kappa_{s w} \alpha}{1+\alpha}\right)^{2}\left(\frac{100}{g}\right)^{\frac{1}{3}} v_{w} S_{s w}(f), \\
S_{s w}(f)= & \left(f / f_{s w}\right)^{3}\left[\frac{7}{4+3\left(f / f_{s w}\right)^{2}}\right]^{\frac{7}{2}},
\end{aligned}
$$

where $\tau_{s w}$ is the duration of the sound wave source, defined as $H_{*} \tau_{s w}=\min \left[1, H_{*} R_{*} / U_{f}\right]$ with $U_{f}$ the root-mean-square fluid velocity [63]

$U_{f} \simeq \frac{\sqrt{3}}{2}\left(\frac{\alpha\left(1-\kappa_{\mathrm{col}}\right)}{1+\alpha\left(1-\kappa_{\mathrm{col}}\right)} \kappa_{s w}\right)^{1 / 2} \stackrel{\alpha \gg 1}{\longrightarrow} \frac{\sqrt{3}}{2}$.

Strong supercooling enhances $U_{f}$, which in turn leads to $H_{*} R_{*} / U_{f}=2 v_{w} /(\sqrt{3} \tilde{\beta}) \lesssim 0.08 \ll 1$ then $\tau_{s w} H_{*} \ll 1$, rendering the sound wave source considerably suppressed. So, in this case, the GW from the sound wave becomes

$$
\begin{aligned}
h^{2} \Omega_{s w}(f)= & 6.3 \times 10^{-5} \frac{1}{\tilde{\beta}^{2}}\left(\frac{\kappa_{s w} \alpha}{1+\alpha}\right)^{2}\left(\frac{100}{g}\right)^{\frac{1}{3}} \\
& \times v_{w}^{2} S_{s w}(f) .
\end{aligned}
$$

For $\alpha \gg 1$, the GW enhancement by strong supercooling is saturated because the explicit $\alpha$ dependence in the spectrum is canceled. Then, the GW spectrum is characterized by the single parameter $\tilde{\beta}$.

(iii) Magnetic hydrodynamics (MHD) turbulence: When the sound wave period ends and the fluid flow becomes nonlinear, percolation generates another fluid bulk motion, the MHD turbulence. If the sound wave period could last over at least one Hubble timescale, then it is supposed to have a suppressed efficiency factor $\kappa_{\text {turb }} \sim 0.05 \kappa_{s w}$ [63]. However, as shown above, $\tau_{s w} \ll 1 / H^{*}$, and then $\kappa_{\text {turb }}$ may be significantly enhanced. But a part of the remaining energy of the bulk fluid motion, namely, the part not consumed by the sound speed wave, can convert into heat, so the quantitative enhancement is unknown yet. Here, we follow Ref. [26], assuming that all of the energy transfers to turbulence, giving rise to the GW spectrum [64]

$$
\begin{aligned}
h^{2} \Omega_{\text {turb }}(f)= & 3.3 \times 10^{-4} \frac{1}{\tilde{\beta}}\left(1-\frac{2(8 \pi)^{1 / 3} v_{w}}{\sqrt{3} \tilde{\beta}}\right) \\
& \times\left(\frac{\kappa_{s w} \alpha}{1+\alpha}\right)^{\frac{3}{2}}\left(\frac{100}{g}\right)^{\frac{1}{3}} v_{w} S_{\text {turb }}(f),
\end{aligned}
$$

with the shape function

$$
S_{\text {turb }}(f)=\frac{\left(f / f_{\text {turb }}\right)^{3}}{\left[1+\left(f / f_{\text {trub }}\right)\right]^{\frac{11}{3}}(1+8 \pi f / h)},
$$

which, compared to $S_{s w}(f)$, shows a moderately large suppression of approximately $\mathcal{O}(10)$ in the high-frequency region. The peak frequency is similar to that of the sound wave source [26]:

$$
\begin{aligned}
f_{\text {turb }}= & 1.65 \times 10^{-5}\left(\frac{T_{n}}{100 \mathrm{GeV}}\right)\left(\frac{g_{*}}{100}\right)^{\frac{1}{6}} \\
& \times \frac{3.9}{\left(v_{w}-c_{s}\right) H_{*} R_{*}} \mathrm{~Hz} \\
= & 3.15 \times 10^{-5} \frac{\tilde{\beta}}{v_{w}}\left(\frac{T_{n}}{100 \mathrm{GeV}}\right)\left(\frac{g_{*}}{100}\right)^{\frac{1}{6}} \mathrm{~Hz}
\end{aligned}
$$

We have to stress that all of these "data-driven" expressions are reliable only for a weaker phase transition $\alpha \lesssim 0.1$. For very large $\alpha$, thus ultra relativistic bubbles, they are far beyond the ability of the current numerical simulation. Recently, there are works toward analytical understanding of the GW in this limiting situation [65].

\section{B. Prospects of the GW signal}

Now, we have collected all the ingredients to demonstrate the tentative prospects of GW signatures of CSIPT by the DM. For an example, in Fig. 8, we show the GW spectra 

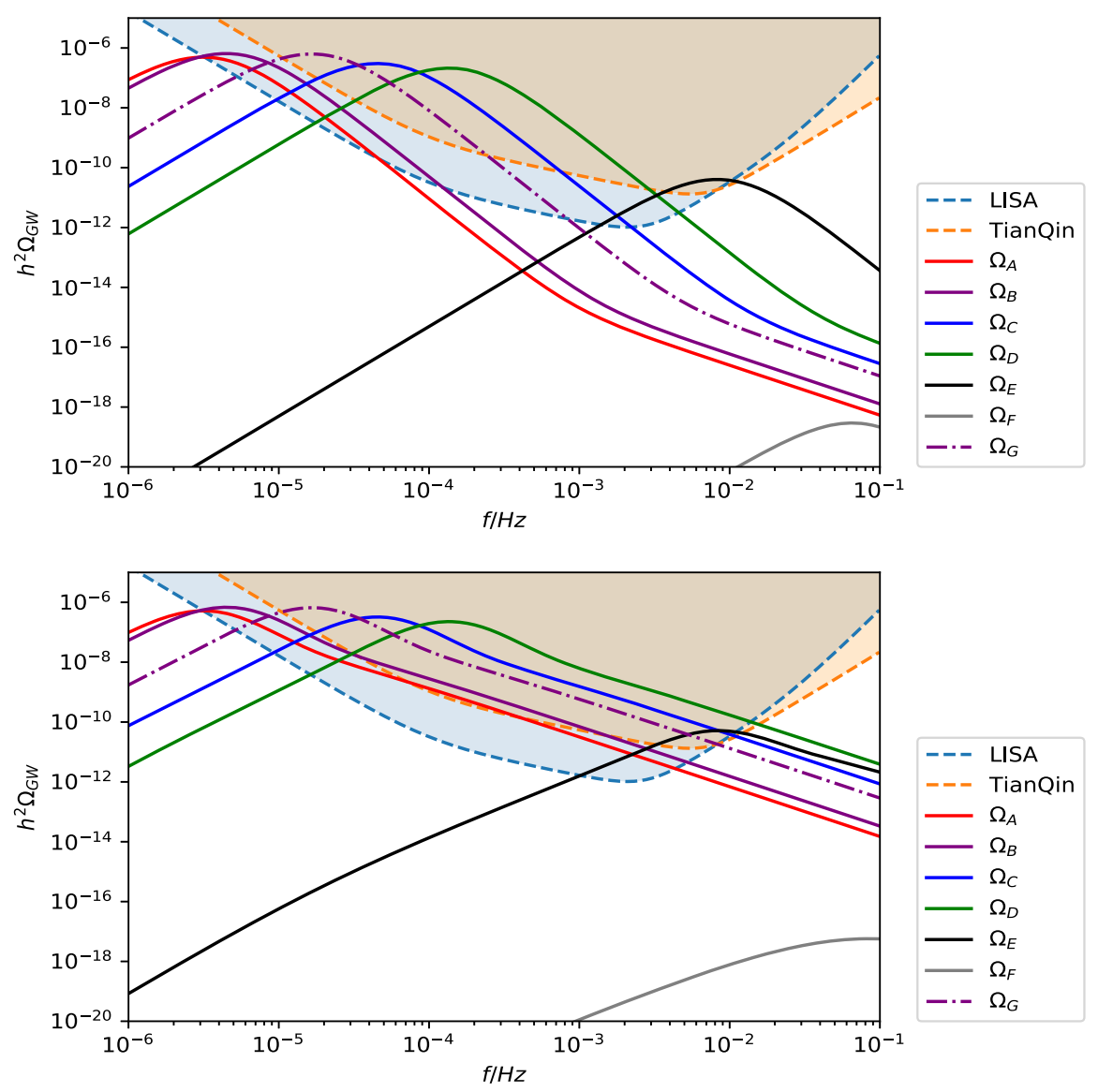

FIG. 8. Detecting prospects of the (total) gravitational wave spectra of the benchmark points listed in Table I, corresponding to the Gildener-Weinberg scenario. For comparison, in the top panel, we include the GW sources from the sound wave and the usual MHD turbulence without enhancement from the shorter duration of the sound wave, while in the bottom panel, we assume that the turbulence is maximally enhanced, which significantly lifts the amplitude at the higher-frequency region.

of the benchmarks given in Table I, for the GW scenario. The sensitivity curves for TianQin and LISA [66,67] are plotted as the boundaries of the shaded regions. Only the spectra of two limiting benchmarks lie below the sensitivity curves. One is $\mathrm{F}$, which has a quite large $\lambda_{s x}$ and hence the resulting bubble nucleation is very effective. As a consequence, the supercooling is weakened, giving rise to a relatively small $\alpha \sim 10^{-2}$. The other one is $\mathrm{A}$, which by contrast has a quite small $\lambda_{s x}$, leading to a very low $T_{n} \sim 1 \mathrm{GeV}$, thus a low peak frequency of approximately $10^{-6} \mathrm{~Hz}$.

Additionally, it is of interest to notice that the DM selfinteraction coupling $\lambda_{x}$, which basically is an irrelevant parameter in the zero-temperature physics, can affect CSIPT through the Daisy term, i.e., $\Pi_{X} \propto \lambda_{x} T^{2}$; see Eq. (4.6). For an illustration, we set up $B$ and $G$ differing only in $\lambda_{x}$. Increasing $\lambda_{x}$, like increasing $\lambda_{s x}$, helps to lift the bubble nucleation rate, thus giving a higher $T_{n}$. Because $\alpha$ is already very large and its dependence in the spectra has been cancelled, then the spectra of B, whose $T_{n}$ is lower then a lower peak frequency, tends to move beyond the sensitivity region.

We end with a comment on the subtle case $\alpha \sim 1$, which is ambiguous to determine the era when CSIPT is completed. We show an example point using two different
PT completion criteria in Table III. Following the crude rule in Sec. IV D, we find that $\alpha$ is very close to 1 using the RD criterion $S(T) \simeq 140$. It indicates that the Universe is transiting from the RD to the vacuum-dominance era, so it should not be a very precise criterion. Then, we also calculate CSIPT taking the vacuum-dominance criterion, and one can see the sharp difference between the resulting PT parameters: $T_{n}$ jumps from $196.1 \mathrm{GeV}$ to $28.6 \mathrm{GeV}$, and as a consequence, the GW spectra significantly shifts to the IR frequency region, i.e., from the gray line to the black line in Fig. 9. The actual CSIPT completion condition is neither $S(T) \simeq 140$ nor $S(T) \simeq 70$ but some value between them, and thus the actual GW spectrum should be located between the two spectra. Unlike in the two limits, in this case, maybe there is no simple condition of $S(T)$ to judge if the CSIPT is completed, but numerically, we can find the condition by using the complete Freedman equation $H(T)^{2}=\left[\rho_{r}(T)+\right.$ $\left.\rho_{0}(T)\right] /\left(3 M_{p l}^{2}\right)$ in the condition like Eq. (4.22). Then, for the given parameters, it is found that $S(T) \approx 118$, between the two limiting values as expected. The resulting accurate PT parameters are shown in Table III, the line labeled as "Mixing." One can see that they are not very different than 


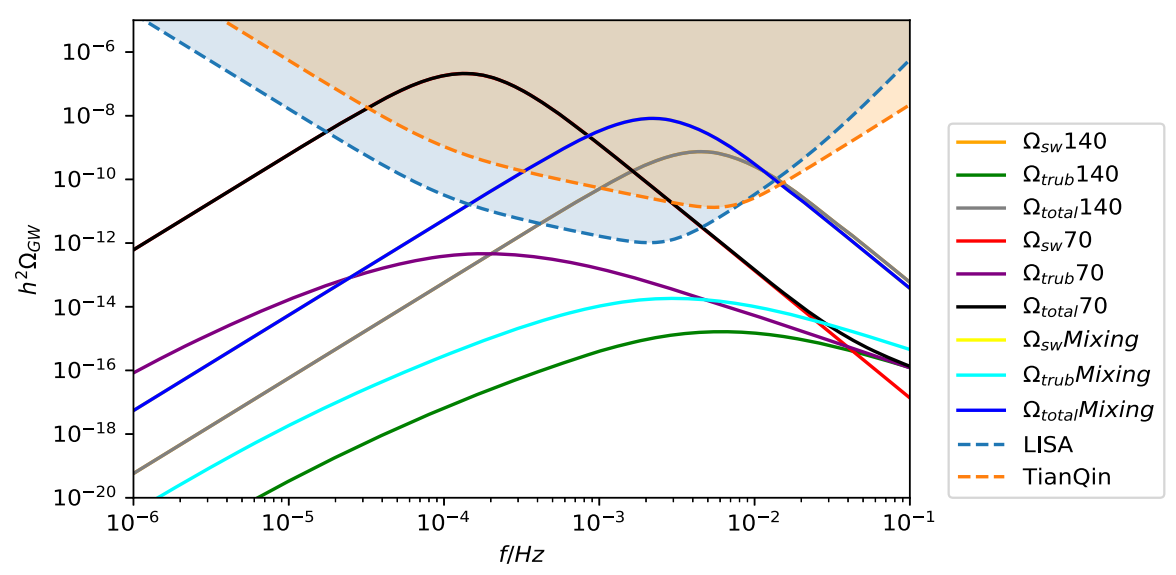

FIG. 9. The gravitational wave spectra from Table III, a subtle case to judge the CSIPT completion era, thus using both criteria for comparison. We display all three sources for the gravitational wave.

those obtained in the RD limit. The blue line in Fig. 9 shows the corresponding GW spectrum.

\section{CONCLUSIONS}

The origin of the weak scale is a fundamental question in the SM. One attractive idea is imposing scale invariance on the classical Lagrangian of some extension to SM, and then a scale is generated at the quantum level due to the anomaly of CSI. But the realistic CSI extension to the SM needs a bosonic trigger, which is assigned to the scalar DM $X$ in this paper. This scenario establishes a direct connection between DM and scale genesis. To accommodate successful DM phenomenologies, the radiative CSISB scale should be approximately $\mathcal{O}(\mathrm{TeV})$, which means that the $\mathrm{GW}$ from CSIPT, with the tendency of a large supercooling, can leave signals at the GW detectors such as LISA and Tianqin. Our analysis of GW signal is based on the usual approach. Recently, Refs. $[68,69]$ presented a substantially improved analysis, which is based on the novel peak-integrated sensitivity curves designed specifically for the GW from the strong first-order PT. If applied, it may enhance detective prospect of our model.

Besides the overall physical picture, we pay great attention to several aspects of techniques that are commonly used despite not being very well understood, summarized in the following:

(i) In Sec. IV B 1, which is based on Gildener-Weinberg scenario, we discuss if the strong quantum corrections could give rise to a significant difference between two analysis methods of CSIPT, which respectively are based on tunneling along the tree-level at direction and full tunneling. Our numerical examples indicate that the tunneling path may be changed but the resulting difference in $S(T)$ is tolerable for normal couplings.

(ii) In Sec. IV B 1, we estimate the quality of Witten's formula, which is frequently used to calculate the nucleation rate in CSIPT, for the one-field case, and find that it is not a very good approximation, owing to the neglect of cubic term in high-temperature expansion. We stress that the essence of Witten's approximation is the observation of validity of hightemperature expansion (to the quartic term) for CSIPT at very low temperature. Furthermore, we argue that it may also apply to the multifield case.

(iii) In Sec. IV C 2, we analyzed the completion condition for CSIPT with a very strong supercooling, which may cause CSIPT completion to occur in the vacuum-dominated era rather than the ordinary RD era. We derive the analytical conditions for $S(T)$ in both cases, taking various criteria.

We are not content with these studies; for instance, a reliable condition for CSIPT in some subtle cases still requires further study.

\section{ACKNOWLEDGMENTS}

We would like to thank Yizhou Lu, Xuenan Chen, and Xiangsong Chen for helpful discussions and especially thank Professor Xiangsong Chen for reading the first edition of this paper. This work is supported in part by the National Science Foundation of China (Grant No. 11775086).

\section{APPENDIX A: ANALYTICAL SOLUTIONS TO RGES}

In this Appendix, we pursue an analytical approximation to Eq. (2.12), which consists of three coupled RGEs. We impose the following hierarchy:

$$
\lambda_{s x} \gg \lambda_{x} \gg \lambda_{s} .
$$

This hierarchy is reasonable. First, $\lambda_{x} \gg \lambda_{s}$ explains why radiative correction drives the $S$ rather than $X$ away from the origin. That is to say, this hierarchy guarantees the stability of DM field $X$. Next, $\lambda_{s x} \gg \lambda_{s}$ is the usual condition for radiative symmetry breaking. Third, since $\lambda_{s x}$ is already relatively large, a smaller $\lambda_{x}$ is good for 
perturbativity. ${ }^{12}$ With the above hierarchy, RGEs in Eq. (2.12) are reduced to a situation similar to scalar QED at the leading order of $\lambda_{x} / \lambda_{s x}$. Then, the solution takes the form of [1]

$$
\begin{aligned}
\lambda_{s x}(t) & =\frac{\lambda_{s x}(0)}{1-\frac{\lambda_{s x}(0)}{8 \pi^{2}} t}, \\
\lambda_{s}(t) & =\frac{b}{2 a} \lambda_{s x}(t)+\frac{X}{a} \lambda_{s x}(t) \tan \left(\frac{X}{b} \log \frac{\lambda_{s x}(t)}{\pi}+A\right),
\end{aligned}
$$

where $t=\log \frac{\mu}{\mu_{0}}$ with $\mu_{0}$ is the renormalization scale; $A=$ $\arctan \frac{a\left[\lambda_{s}(0)-\frac{b}{2 a} \lambda_{s x}(0)\right]}{X \lambda_{s x}(0)}-\frac{X}{b} \log \frac{\lambda_{s x}(0)}{\pi}$ with $X=\sqrt{a c-\frac{b^{2}}{4}}$ in which the constants $a=\frac{27}{2 \pi}, b=\frac{1}{4 \pi}, c=\frac{9}{8 \pi}$.

\footnotetext{
${ }^{12}$ In principle, we have no compelling arguments to exclude the opposite pattern $\lambda_{x} \gg \lambda_{s x}$. Actually, it is of interest to explore if radiative symmetry breaking can be driven by a large DM selfinteraction $\lambda_{x}$, basically a two-loop effect on $\lambda_{s}$. Such a scenario has not been discussed yet.
}

\section{APPENDIX B: THE FAILURE OF COMMON HIGH-TEMPERATURE EXPANSION}

In this Appendix, we give an example to show that in analyzing CSIPT the usual high-temperature expansion keeping only the quadratic term [34,70] may lead to a sizable error in calculating $S(T)$. We consider the GildenerWeinberg scenario of our model, and the effective potential in the high-temperature expansion to the quadratic term is given by

$$
\begin{aligned}
V_{H T}(\phi, T)= & V_{0}(\phi)+V_{0}^{(1)}(\phi)+C T^{2} \phi^{2}, \\
C= & \frac{1}{12\langle\phi\rangle}\left(m_{\phi_{1}}^{2}(\vec{n})+m_{X}^{2}(\vec{n})+6 m_{W}^{2}(\vec{n})\right. \\
& \left.+3 m_{Z}^{2}(\vec{n})+6 m_{t}^{2}(\vec{n})\right) .
\end{aligned}
$$

Our numerical example is the point $\mathrm{E}$ in Table I. Using $V_{T H}$, we obtain that the CSIPT completion temperature is $18 \mathrm{GeV}$, in the vacuum-dominated era. By contrast, the CSIPT is found to be completed in the RD era, at $278 \mathrm{GeV}$, if we use the complete effective potential. Therefore, the wrongly used high temperature may lead to dramatic difference in CSIPT.
[1] S. R. Coleman and E. J. Weinberg, Phys. Rev. D 7, 1888 (1973).

[2] W. A. Bardeen, Report No. FERMILAB-CONF-95-391-T; Report No. C95-08-27.3, 1995.

[3] T. Hur, D. W. Jung, P. Ko, and J. Y. Lee, Phys. Lett. B 696, 262 (2011).

[4] J. Guo, Z. Kang, P. Ko, and Y. Orikasa, Phys. Rev. D 91, 115017 (2015).

[5] J. Guo and Z. Kang, Nucl. Phys. B898, 415 (2015).

[6] Z. Kang, Eur. Phys. J. C 75, 471 (2015); Phys. Lett. B 751, 201 (2015).

[7] Y. Ametani, M. Aoki, H. Goto, and J. Kubo, Phys. Rev. D 91, 115007 (2015).

[8] H. Ishida, S. Matsuzaki, and Y. Yamaguchi, Prog. Theor. Exp. Phys. 2017, 103B01 (2017).

[9] T. Hambye, A. Strumia, and D. Teresi, J. High Energy Phys. 08 (2018) 188.

[10] C. Guo, S. Y. Guo, and Y. Liao, Chin. Phys. C 43, 103102 (2019).

[11] L. Bian, W. Cheng, H. K. Guo, and Y. Zhang, arXiv:1907 .13589 .

[12] D. W. Jung, J. Lee, and S. H. Nam, Phys. Lett. B 797, 134823 (2019).

[13] A. Karam and K. Tamvakis, Phys. Rev. D 92, 075010 (2015).

[14] V. Brdar, A. J. Helmboldt, and J. Kubo, J. Cosmol. Astropart. Phys. 02 (2019) 021.
[15] L. Chataignier, T. Prokopec, M. G. Schmidt, and B. Świeżewska, J. High Energy Phys. 08 (2018) 083.

[16] E. Gildener and S. Weinberg, Phys. Rev. D 13, 3333 (1976).

[17] R. Hempfling, Phys. Lett. B 379, 153 (1996); K. A. Meissner and H. Nicolai, Phys. Lett. B 648, 312 (2007); W.-F. Chang, J. N. Ng, and J. M. S. Wu, Phys. Rev. D 75, 115016 (2007); C. Englert, J. Jaeckel, V. V. Khoze, and M. Spannowsky, J. High Energy Phys. 04 (2013) 060.

[18] C. W. Chiang and E. Senaha, Phys. Lett. B 774, 489 (2017).

[19] V. Brdar, A. J. Helmboldt, and M. Lindner, J. High Energy Phys. 12 (2019) 158.

[20] M. Aoki and J. Kubo, J. Cosmol. Astropart. Phys. 04 (2020) 001.

[21] A. Mohamadnejad, Eur. Phys. J. C 80, 197 (2020).

[22] C. Marzo, L. Marzola, and V. Vaskonen, Eur. Phys. J. C 79, 601 (2019).

[23] J. Kubo and M. Yamada, J. Cosmol. Astropart. Phys. 12 (2016) 001.

[24] E. Witten, Nucl. Phys. B177, 477 (1981).

[25] T. Prokopec, J. Rezacek, and B. wieewska, J. Cosmol. Astropart. Phys. 02 (2019) 009.

[26] J. Ellis, M. Lewicki, J. M. No, and V. Vaskonen, J. Cosmol. Astropart. Phys. 06 (2019) 024.

[27] R. Hempfling, Phys. Lett. B 379, 153 (1996); W.-F. Chang, J. N. Ng, and J. M. S. Wu, Phys. Rev. D 75, 115016 (2007); S. Iso, N. Okada, and Y. Orikasa, Phys. Rev. D 80, 115007 
(2009); M. Holthausen, M. Lindner, and M. A. Schmidt, Phys. Rev. D 82, 055002 (2010).

[28] S. Yaser Ayazi and A. Mohamadnejad, J. High Energy Phys. 03 (2019) 181.

[29] C. D. Carone and R. Ramos, Phys. Rev. D 88, 055020 (2013).

[30] T. Hambye and A. Strumia, Phys. Rev. D 88, 055022 (2013).

[31] A. Karam and K. Tamvakis, Phys. Rev. D 94, 055004 (2016).

[32] D. Chway, T. H. Jung, H. D. Kim, and R. Dermisek, Phys. Rev. Lett. 113, 051801 (2014).

[33] A. Farzinnia, H. J. He, and J. Ren, Phys. Lett. B 727, 141 (2013).

[34] P. H. Ghorbani, Phys. Rev. D 98, 115016 (2018).

[35] M. Lindner, S. Schmidt, and J. Smirnov, J. High Energy Phys. 10 (2014) 177; A. Ahriche, K. L. McDonald, and S. Nasri, J. High Energy Phys. 02 (2016) 038; T. Nomura, H. Okada, and Y. Orikasa, Phys. Rev. D 94, 115018 (2016).

[36] E. Gildener, Phys. Rev. D 13, 1025 (1976).

[37] A. Andreassen, W. Frost, and M. D. Schwartz, Phys. Rev. D 91, 016009 (2015).

[38] A. Andreassen, W. Frost, and M. D. Schwartz, Phys. Rev. Lett. 113, 241801 (2014).

[39] F. Loebbert, J. Miczajka, and J. Plefka, Phys. Rev. D 99, 015026 (2019).

[40] M. Quiros, arXiv:hep-ph/9901312.

[41] G. Jungman, M. Kamionkowski, and K. Griest, Phys. Rep. 267, 195 (1996).

[42] X. Gao, Z. Kang, and T. Li, J. Cosmol. Astropart. Phys. 01 (2013) 021.

[43] E. Aprile et al. (XENON Collaboration), Phys. Rev. Lett. 119, 181301 (2017).

[44] S. Weinberg, Phys. Rev. D 9, 3357 (1974).

[45] L. Dolan and R. Jackiw, Phys. Rev. D 9, 3320 (1974).

[46] D. J. Gross, R. D. Pisarski, and L. G. Yaffe, Rev. Mod. Phys. 53, 43 (1981).

[47] D. Curtin, P. Meade, and H. Ramani, Eur. Phys. J. C 78, 787 (2018).

[48] S. Coleman, Phys. Rev. D 15, 2929 (1977); C. G. Callan and S. Coleman, Phys. Rev. D 16, 1762 (1977).

[49] A. D. Linde, Phys. Lett. 70B, 306 (1977); 100B, 37 (1981); Nucl. Phys. B216, 421 (1983).

[50] C. L. Wainwright, Comput. Phys. Commun. 183, 2006 (2012).
[51] R. Jinno and M. Takimoto, Phys. Rev. D 95, 015020 (2017).

[52] E. Brczin and G. Parisi, J. Stat. Phys. 19, 269 (1978).

[53] J. Ellis, M. Lewicki, and J. M. No, J. Cosmol. Astropart. Phys. 04 (2019) 003.

[54] A. H. Guth, Phys. Rev. D 23, 347 (1981).

[55] E. Witten, Nucl. Phys. B177, 477 (1981); S. Iso, P. D. Serpico, and K. Shimada, Phys. Rev. Lett. 119, 141301 (2017).

[56] M. S. Turner, E. J. Weinberg, and L. M. Widrow, Phys. Rev. D 46, 2384 (1992).

[57] A. H. Guth and H. Tye, Phys. Rev. Lett. 44, 631 (1980); 44, 963(E) (1980).

[58] A. H. Guth and E. J. Weinberg, Nucl. Phys. B212, 321 (1983).

[59] D. Bodeker and G. D. Moore, J. Cosmol. Astropart. Phys. 05 (2017) 025.

[60] J. R. Espinosa, T. Konstandin, J. M. No, and G. Servant, J. Cosmol. Astropart. Phys. 06 (2010) 028.

[61] C. Caprini, M. Hindmarsh, S. Huber, T. Konstandin, J. Kozaczuk, G. Nardini, J. M. No, A. Petiteau, P. Schwaller, G. Servant, and D. J. Weir, J. Cosmol. Astropart. Phys. 04 (2016) 001.

[62] C. Caprini, M. Chala, G. C. Dorsch, M. Hindmarsh, S. J. Huber, T. Konstandin, J. Kozaczuk, G. Nardini, J. M. No, K. Rummukainen, P. Schwaller, G. Servant, A. Tranberg, and D. J. Weir, J. Cosmol. Astropart. Phys. 03 (2020) 024.

[63] M. Hindmarsh, S. J. Huber, K. Rummukainen, and D. J. Weir, Phys. Rev. D 92, 123009 (2015).

[64] C. Caprini, R. Durrer, and G. Servant, J. Cosmol. Astropart. Phys. 12 (2009) 024.

[65] R. Jinno, H. Seong, M. Takimoto, and C. M. Um, J. Cosmol. Astropart. Phys. 10 (2019) 033.

[66] C. J. Moore, R. H. Cole, and C. P. L. Berry, Classical Quantum Gravity 32, 015014 (2015).

[67] Y. Lu, Y. Gong, Z. Yi, and F. Zhang, J. Cosmol. Astropart. Phys. 12 (2019) 031.

[68] T. Alanne, T. Hugle, M. Platscher, and K. Schmitz, J. High Energy Phys. 03 (2020) 004.

[69] K. Schmitz, arXiv:2002.04615.

[70] L. Marzola, A. Racioppi, and V. Vaskonen, Eur. Phys. J. C 77, 484 (2017). 